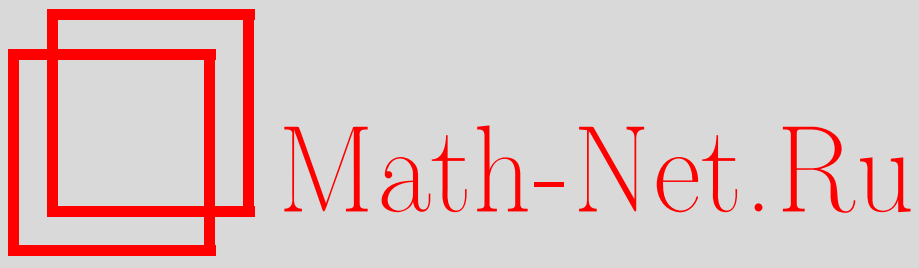

С. С. Платонов, Инвариантные подпространства в некоторых функциональных пространствах на симметрических пространствах. II, Изв. РАН. Сер. матем., 1998, том 62, выпуск 2, 131-168

DOI: https://doi.org/10.4213/im178

Использование Общероссийского математического портала Math-Net.Ru подразумевает, что вы прочитали и согласны с пользовательским соглашением http://www . mathnet.ru/rus/agreement

Параметры загрузки:

IP : 3.85 .7 .115

26 апреля 2023 г., 14:11:47 
УДК 517.954

\title{
С. С. Платонов \\ Инвариантные подпространства \\ в некоторых функциональных пространствах на симметрических пространствах. II
}

\begin{abstract}
Пусть $G$ - полупростая связная группа Ли с конечным центром, $K$ - максимальная компактная подгруппа в $G, M=G / K$ - риманово симметрическое пространство некомпактного типа. Изучается задача об описании строения линейных замкнутых подпространств в различных функциональных топологических векторных пространствах на $M$, инвариантных относительно квазирегулярного представления группы $G$. Рассмотрен случай, когда $M$ - симплектическое симметрическое пространство ранга 1.
\end{abstract}

Библиография: 17 наименований.

\section{§ 1. Введение и формулировка основных результатов}

Пусть группа $G$ транзитивно действует на гладком многообразии $M$. Для $g \in G$ и любой функции $f(x)$ на $M$ положим

$$
(\pi(g) f)(x)=f\left(g^{-1} x\right)
$$

Локально выпуклое пространство (ЛВП) $\mathscr{F}$, состоящее из комплекснозначных функций на $M$ (обычных или обобщенных), будем называть $\pi$-инвариантнылм, если из $f(x) \in \mathscr{F}$ и $g \in G$ следует, что $\pi(g) f \in \mathscr{F}$ и отображение $g \rightarrow \pi(g) f$ из $G$ и $\mathscr{F}$ непрерывно. В этом случае операторы $\left.\pi(g)\right|_{\mathscr{F}}$ (будем обозначать их просто через $\pi(g))$ определяют квазирегулярное представление группы $G$ в топологическом векторном пространстве $\mathscr{F}$. Линейное подпространство $H \subseteq \mathscr{F}$ будем называть инвариантным подпространством (ИПП), если оно замкнуто и $\pi$-инвариантно. Одной из основных задач гармонического анализа на группах Ли является задача об описании всех ИПП для конкретных групп Ли и конкретных функциональных пространств, выделяемых некоторыми условиями гладкости и роста (см. [1], где перечислены некоторые результаты об описании ИПП).

Далее будем рассматривать случай, когда $M=G / K$ - риманово симметрическое пространство некомпактного типа, $G$ - полупростая связная группа Ли с конечным центром, $K$ - максимальная компактная подгруппа в группе $G$. Группа $G$ транзитивно действует на $M$ левыми сдвигами. Риманова метрика на $M=$ $G / K$ индуцируется формой Киллинга на алгебре Ли $\mathfrak{g}_{0}$ групшы $G$. В качестве

Работа поддержана РФФИ, проект 95-01-01391.

$$
\text { (C) С.С. ПлАтонов, } 1998
$$


функциональных пространств $\mathscr{F}$ будем рассматривать пространства двух классов. Пространства одного класса состоят из "всех" функций на $M$, удовлетворяющих некоторым условиям гладкости. Этому классу принадлежат, например, пространства $d$ раз непрерывно дифференцируемых функций $C^{d}(M)(d=0,1,2, \ldots, \infty)$, в частности пространства $C^{0}(M)=C(M)$ непрерьвных функций и $C^{\infty}(M)=$ $\mathscr{E}(M)$ бесконечно дифференцируемых функций (все пространства берутся с обычными топологиями). Более точно: пусть $\mathscr{D}$ - пространство бесконечно дифференцируемых функций на $M$ с компактным носителем, $\mathscr{D}^{\prime}$ - пространство обобщенных функций на $M$, тогда в качестве $\mathscr{F}$ можно взять любое полное ЛВП такое, что

$$
\mathscr{E} \subseteq \mathscr{F} \subseteq \mathscr{D}^{\prime}
$$

(вложения предполагаются непрерывными). Будем называть такие пространства $\mathscr{F}$ пространствами типа 1.

Другие функциональные пространства состоят из функций экспоненциального роста в некотором смысле, который нуждается в уточнении. Пусть $o=\mathrm{e} K-$ фиксированная точка пространства $M=G / K$ (е-единичный элемент в группе $G$ ). Для $x \in M$ пусть $|x|$ - расстояние от точки $x$ до точки $o$. Обозначим через $C_{k}, k \in \mathbb{R}$, пространство непрерывных функций $f(x)$ на $M$, для которых $|f(x)| e^{-k|x|} \rightarrow 0$ при $|x| \rightarrow \infty$. Пространство $C_{k}$ является банаховым пространством (БП) относительно нормы

$$
N_{k}(f)=\sup _{x \in M}|f(x)| e^{-k|x|} .
$$

Пространство

$$
C_{*}=\bigcup_{k>0} C_{k}
$$

снабдим топологией индуктивного предела БП $C_{k}$.

Пусть $\mathfrak{g}_{0}$ - алгебра Ли группы $G$. Для $X \in \mathfrak{g}_{0}$ и функции $f(x)$ пусть

$$
(X f)(x)=\left.\frac{d}{d t} f(\exp (-t X) x)\right|_{t=0}
$$

т.е. действие $X f$ индуцировано действием (1.1) группы $G$. Выберем какой-нибудь базис $X_{1}, \ldots, X_{m}$ в $\mathfrak{g}_{0}$. Через $\mathscr{E}_{k}$ обозначим множество функций $f(x) \in C^{\infty}(M)$, которые принадлежат пространству $C_{k}$ вместе со всеми производными, т.е.

$$
X_{i_{1}} \ldots X_{i_{r}} f \in C_{k}
$$

для любых векторов $X_{i_{1}}, \ldots, X_{i_{r}}$ из базиса. Пространство $\mathscr{E}_{k}$ является локально выпуклым пространством с топологией, порожденной семейством полунорм $N_{k, d}$, $d \in \mathbb{Z}_{+}=\{0,1,2, \ldots\}$, где

$$
N_{k, d}(f)=\sum N_{k}\left(X_{i_{1}} \ldots X_{i_{r}} f\right),
$$


суммирование происходит по всевозможным наборам $\left(i_{1}, \ldots, i_{r}\right), 0 \leqslant r \leqslant d$, каждый индекс в наборе пробегает значения от 1 до $m$.

Пространство

$$
\mathscr{E}_{*}=\bigcup_{k>0} \mathscr{E}_{k}
$$

снабдим топологией индуктивного предела ЛВП $\mathscr{E}_{k}$.

Пусть

$$
\mathscr{K}=\bigcap_{k>0} \mathscr{E}_{-k} .
$$

Пространство $\mathscr{K}$ состоит из функций, которые стремятся к нулю со всеми производными быстрее, чем $e^{-k|x|}$ для любого $k>0$. Пространство $\mathscr{K}$ является ЛВП с топологией, порожденной системой полунорм $N_{-k, d}$ для всех $k>0, d \in \mathbb{Z}_{+}$. Пусть $\mathscr{K}^{\prime}$ - сопряженное к $\mathscr{K}$ пространство, снабженное слабой топологией. $\mathscr{K}^{\prime}$ естественно назвать пространством обобщенных функиий экспоненциального роста.

Будем называть полное $\pi$-инвариантное пространство $\mathscr{F}$ функииональныл м пространством әкспоненциального роста или пространством типа 2 , если

$$
\mathscr{E}_{*} \subseteq \mathscr{F} \subseteq \mathscr{K}^{\prime}
$$

(вложения предполагаются непрерывными).

Примерами пространств типа 2 являются пространства $C_{*}$ и $\mathscr{E}_{*}$. Другие примеры пространств типа 2 приведены в $[1, \S 2]$.

Задачи об описании строения ИПП в функциональных пространствах на симметрических пространствах (особенно в пространстве $C^{\infty}(M)$ ) тесно связаны с задачами о спектральном синтезе на симметрических пространствах, о структуре решений уравнений и систем уравнений в свертках и с проблемой Помпейю на симметрических пространствах (см. [2]-[5]). Для симметрических пространств ранга $\geqslant 2$ пока нет подходов к описанию строения ВСЕХ ИПП и, по-видимому, эта задача очень сложная (см. [3]), хотя удается получить описание строения некоторых важных ИПП в $C^{\infty}(M)$, которые являются пространствами решений инвариантных дифференциальных уравнений и уравнений в свертках на $M$ (см. [6], [7]).

Настояшая работа является продолжением серии статей, в которых получено полное описание ИПП в функциональных пространствах типов 1 и 2 на симметрических пространствах ранга 1 некомпактного типа. Из классификации симметрических пространств (см. [8]) известно, что все римановы симметрические пространства $G / K$ ранга 1 некомпактного типа исчерпываются тремя бесконечными сериями (всюду $n \geqslant 2): \mathrm{SO}_{0}(n, 1) / \mathrm{SO}(n), \mathrm{SU}(1, n) / U(n), \mathrm{Sp}(1, n) / \mathrm{Sp}(1) \times \mathrm{Sp}(n)$, и одним особым симметрическим пространством (пространством Кэли).

Пусть $\Lambda$ - множество классов эквивалентности неприводимых конечномерных представлений группы $K$, или, эквивалентно, множество старших весов неприводимых представлений, так как любое неприводимое представление групшы $K$ задается своим старшим весом. Для $\lambda \in \Lambda$ пусть $T^{\lambda}(u)$ - соответствующее неприводимое представление группы $K, E^{\lambda}$ - пространство представления, $\langle$,$\rangle - некото-$ рая $K$-инвариантная эрмитова форма в $E^{\lambda}$. 
Если $\mathscr{F}$ - произвольное ЛВП, состоящее из функций на $M, E$ - конечномерное нормированное пространство над $\mathbb{C}$, то тензорное произведение векторных пространств $\mathscr{F} \otimes E$ естественным образом отождествляется с пространством функций на $M$ со значениями в $E$ и снабжается топологией тензорного произведения ЛВП [9]. Через $\mathscr{F}(\lambda)$ обозначим множество функций $F \in \mathscr{F} \otimes E^{\lambda}$, удовлетворяюших условию

$$
F(u x)=T^{\lambda}(u) F(x) \quad \forall u \in K .
$$

Подпространство $\mathscr{F}(\lambda)$ замкнуто в $\mathscr{F} \otimes E^{\lambda}$ и снабжается индуцированной топологией. В частности, возникают пространства $C^{d(\lambda)}$ и $\mathscr{E}(\lambda)=C^{\infty(\lambda)}$. Пусть $\Lambda_{0}-$ множество тех $\lambda \in \Lambda$, для которых $\mathscr{F}^{(\lambda)} \neq\{0\}$.

Для любого ИПП $H \subset \mathscr{F}$ (везде будем считать, что $H \neq \mathscr{F}$ ) через $H^{(\lambda)}$ обозначим множество всех функций $F(x) \in \mathscr{F}^{(\lambda)}$ таких, что для всякого $\xi \in E^{\lambda}$ функция $f_{\xi}(x)=\langle F(x), \xi\rangle \in H$. Если $\mathscr{F}$ - полное ЛВП, то подпространство $H$ однозначно восстанавливается по набору подпространств $H^{(\lambda)}$, а именно $H$ совпадает с замыканием в $\mathscr{F}$ линейной оболочки функций $\langle F(x), \xi\rangle$ при $F \in H^{(\lambda)}, \xi \in E^{\lambda}, \lambda \in \Lambda$ (см. $[1, \S 3])$. Будем называть подпространства $H^{(\lambda)}$ ячейками ИПП $H$ или просто ячейками. Ненулевые ячейки могут быть только при $\lambda \in \Lambda_{0}$.

Обшая схема описания ИПП следуюшая: описывается строение всевозможных ячеек и затем определяются условия, при которых набор ячеек соответствует одному ИПП. Для симметрических пространств $\mathrm{SO}_{0}(n, 1) / \mathrm{SO}(n)$ и $\mathrm{SU}(1, n) / U(n)$ такое описание ИПП получено в [1], [10], [11]. В настоящей статье описаны ИПП для $M=\mathrm{Sp}(1, n) / \mathrm{Sp}(1) \times \mathrm{Sp}(n)$.

В дальнейшем, если не оговорено противное, будем предполагать, что $M=$ $\operatorname{Sp}(1, n) / \operatorname{Sp}(1) \times \operatorname{Sp}(n), G=\operatorname{Sp}(1, n), K=\operatorname{Sp}(1) \times \operatorname{Sp}(n)$. Пусть $\Delta-$ оператор Лапласа-Бельтрами на симметрическом пространстве $M, \tilde{\Delta}=8(n+2) \Delta$. Для $\mu \in \mathbb{C}$ и натурального $r$ через $V_{\mu, r}^{(\lambda)}$ обозначим подпространство в $\mathscr{E}(\lambda)$, состоящее из всех функций $F(x)$, удовлетворяюших дифференциальному уравнению

$$
\left(\tilde{\Delta}+\mu^{2}+\rho^{2}\right)^{r} F=0, \quad \rho=2 n+1 .
$$

Далее будет показано, что при $\lambda \in \Lambda_{0} \operatorname{dim} V_{\mu, r}^{(\lambda)}=r$ и в пространстве $V_{\mu, r}^{(\lambda)}$ можно выбрать жорданов базис, т.е. такой базис $F_{1}, \ldots, F_{r}$, что $\tilde{\Delta} F_{1}=-\left(\mu^{2}+\rho^{2}\right) F_{1}$, $\tilde{\Delta} F_{k}=-\left(\mu^{2}+\rho^{2}\right) F_{k}+F_{k-1}$ для $k \geqslant 2$.

Для всех рассматриваемых пространств $\mathscr{F}$ подпространство $V_{\mu, r}^{(\lambda)}$ содержится в $\mathscr{F}(\lambda)$. Если обозначить

$$
\mathbb{C}_{+}=\{z \in \mathbb{C}: \operatorname{Re} z \geqslant 0 \text { и при } \operatorname{Re} z=0 \operatorname{Im} z \geqslant 0\},
$$

то без ограничения обшности можно считать, что $\mu \in \mathbb{C}_{+}$.

Подпространство $V_{\mu, r}^{(\lambda)}$ является простейшей ячейкой в $\mathscr{F}(\lambda)$. В общем случае строение ячеек в $\mathscr{F}^{(\lambda)}$ описывается следуюшей теоремой. 
Teорема I. Для любой ячейки $H^{(\lambda)}$ в $\mathscr{F}(\lambda)\left(\lambda \in \Lambda_{0}\right)$ cуществует единственный конечный или счетный набор комплексных чисел $\sigma\left(\sigma \subset \mathbb{C}_{+}\right.$и каждое число может входить в $\sigma$ с конечной кратностью) такой, что $H^{(\lambda)}$ является замыканием в $\mathscr{F}^{(\lambda)}$ линейной оболочки подпространств $V_{\mu, r}^{(\lambda)}$, где $\mu$ пробегает набор $\sigma$, а $r$ - кратность иисла $\mu$ в әтом наборе.

Естественно назвать набор $\sigma$ спектром ячейки $H^{(\lambda)}$. Можно получить и описание спектров ячеек. Для пространства $\mathscr{F}$ типа 1 (типа 2) всевозможные спектры совпадают с наборами, удовлетворяюшими следуюшему условию $\left(A_{1}\right)$ (соответственно условию $\left.\left(A_{2}\right)\right)$.

$\left(A_{1}\right)$ Существует челая ненулевая функиия $\Phi(z), \quad z=\xi+i \eta$, для которой каждое число $\mu$, входящее в набор $\sigma$ с кратностью $r$, является корнем функиии $\Phi(z)$ кратности $r$, причем

$$
|\Phi(z)| \leqslant A e^{B|\eta|}(1+|z|)^{C}
$$

для некоторых $A, B, C>0$ (такие функиии $\Phi(z)$ являются преобразованиями Фурье обобщенных функиий с компактными носителями).

$\left(A_{2}\right)$ При каждом $t>0$ набор $\sigma_{t}=\{\mu \in \sigma: \operatorname{Re} \mu \leqslant t\}$ должен быть конечным или, после упорядочения чисел из $\sigma_{t}$ в порядке возрастания $\operatorname{Im} \mu$ $\left(0 \leqslant \operatorname{Im} \mu_{1} \leqslant \operatorname{Im} \mu_{2} \leqslant \cdots\right)$, должно выполняться условие $\operatorname{Im} \mu_{j} / j \rightarrow \infty$.

Пусть $\mathfrak{k}_{0}$ - алгебра Ли группы $K, \mathfrak{k}$ - комплексификация алгебры $\mathfrak{k}_{0}$. В нашем случае группа $K$ является полупростой и односвязной, поэтому неприводимые представления групшы $K$ отождествляются с конечномерными неприводимыми представлениями соответствуюшей полупростой алгебры k. Так как каждое неприводимое конечномерное представление алгебры Ли k однозначно определяется своим старшим весом, то множество $\Lambda$ отождествляется с множеством старших весов алгебры Ли k.

Пусть $\mathfrak{k}$ - произвольная полупростая алгебра Ли над $\mathbb{C}, \mathfrak{h}$ - ее подалгебра Картана. Если $\alpha$ - корень алгебры $\mathfrak{k}, \mathfrak{k}(\alpha)$ - соответствующее ему корневое подпространство, то $[\mathfrak{k}(\alpha), \mathfrak{k}(-\alpha)]$ - одномерное подпространство в $\mathfrak{h}$. В этом подпространстве существует единственный вектор $H_{\alpha}$ такой, что $\alpha\left(H_{\alpha}\right)=2$ (необходимые сведения из теории полупростых алгебр Ли см. в [12] или [13]). Пусть $\alpha_{1}, \ldots, \alpha_{m}$ - система простых корней алгебры k. Любое неприводимое конечномерное представление алгебры Ли $\mathfrak{k}$ задается старшим весом $\lambda \in \mathfrak{h}^{*}\left(\mathfrak{h}^{*}-\right.$ множество линейных функционалов на $\mathfrak{h})$. При этом линейный функционал $\lambda \in \mathfrak{h}^{*}$ является старшим весом некоторого неприводимого конечномерного представления алгебры Ли k тогда и только тогда, когда все числа $\lambda_{j}=\lambda\left(H_{\alpha_{j}}\right) \in \mathbb{Z}_{+}$. Такие линейные функционалы называются доминантными. Поэтому будем отождествлять старший вес $\lambda$ с набором неотрицательных целых чисел $\lambda=\left(\lambda_{1}, \ldots, \lambda_{m}\right)$, где $\lambda_{j}=\lambda\left(H_{\alpha_{j}}\right)$.

В нашем случае алгебра Ли $\mathfrak{k}=\operatorname{sp}(1) \times \operatorname{sp}(n), n \geqslant 2$. Выберем простые корни $\alpha_{0}, \alpha_{1}, \ldots, \alpha_{n}$ алгебры Ли k так, что $\alpha_{0}$ - корень подалгебры $\mathrm{sp}(1)$, a $\alpha_{1}, \ldots, \alpha_{n}-$ 
простые корни подалгебры $\mathrm{sp}(1)=C_{n}$, взятые в обычном порядке, который определяется, например, диаграммой Дьнкина

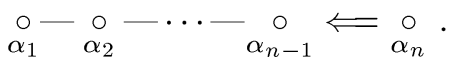

В этом случае множество $\Lambda_{0}$ состоит из старших весов $\lambda=\left(\lambda_{0}, \lambda_{1}, \ldots, \lambda_{n}\right)$ алгебры $\mathfrak{k}$, имеющих вид $\lambda=(k, k, l, 0, \ldots, 0)$, где $k, l \in \mathbb{Z}_{+}$(т.е. $\lambda_{0}=\lambda_{1}=k, \lambda_{2}=l$, $\left.\lambda_{3}=\cdots=\lambda_{n}=0\right)$.

Пусть в каждом пространстве $\mathscr{F}^{(\lambda)}, \lambda \in \Lambda_{0}$, зафиксирована ячейка $H^{(\lambda)}$ некоторого ИПП, вообще говоря, зависящего от $\lambda$, и пусть $\sigma(\lambda)-$ спектр ячейки $H^{(\lambda)}$. Следующая теорема дает необходимые и достаточные условия для того, чтобы ячейки $H^{(\lambda)}$ были ячейками одного ИПП. Предварительно введем целочисленные векторы $\delta_{1}, \delta_{2} \in \mathbb{Z}^{n+1}$ и зависящие от $\lambda$ числа $\mu_{1}(\lambda), \mu_{2}(\lambda) \in \mathbb{C}$. Пусть $\delta_{1}=(1,1,0, \ldots, 0), \quad \delta_{2}=(-1,-1,1,0, \ldots, 0), \quad \lambda=(k, k, l, 0, \ldots, 0) \in \Lambda_{0}$, $\mu_{1}(\lambda)=i(2 k+2 l+2 n+1), \quad \mu_{2}(\lambda)=i(2 l+2 n-1)$, где $i=\sqrt{-1}$.

Теорема II. Ячейки $H^{(\lambda)}, \lambda \in \Lambda_{0}$, соответствуют одному ИПП тогда и только тогда, когда выполняются условия:

1) для любого $\lambda \in \Lambda_{0}$ спектры $\sigma(\lambda)$ и $\sigma\left(\lambda+\delta_{1}\right)$ должнны совпадать или спектр $\sigma\left(\lambda+\delta_{1}\right)$ получается из $\sigma(\lambda)$ уменьшением кратности числа $\mu_{1}(\lambda)$ на 1 ;

2) если $\lambda \in \Lambda_{0} u \lambda+\delta_{2} \in \Lambda_{0}$, то спектры $\sigma(\lambda) u \sigma\left(\lambda+\delta_{2}\right)$ должниь совпадать или спектр $\sigma\left(\lambda+\delta_{2}\right)$ получается из $\sigma(\lambda)$ уменьшением кратности числа $\mu_{2}(\lambda)$ на 1 .

В совокупности теоремы I и II дают полное описание инвариантных подпространств в $\mathscr{F}$, и их доказательство является основной целью настоящей работы. Используемые при этом методы близки к методам работ [1], [10], [11], но имеются и существенные отличия. Основное отличие состоит в том, что для симметрических пространств $\mathrm{SO}_{0}(1, n) / \mathrm{SO}(n)$ и $\mathrm{SU}(1, n) / U(n)$ в процессе вычислений существенно использовались базис Гельфанда-Цетлина в пространствах $E^{\lambda}$ неприводимых представлений группы $K(K=\mathrm{SO}(n)$ или $K=U(n))$ и явные формулы действия алгебры Ли группы $K$ в этом базисе. Для симметрического пространства $\operatorname{Sp}(1, n) / \operatorname{Sp}(1) \times \operatorname{Sp}(n)$ в пространствах $E^{\lambda}$ приходится использовать весовой базис, что значительно усложняет вычисления (см. §4).

Из теорем I и II легко получить описание неприводимых и неразложимых ИПП в $\mathscr{F}$, где ИПП $H$ называется неприводимым. , если в $H$ нет собственных ИПП, и $H$ называется неразложимым, если $H \neq H_{1}+H_{2}$ для ненулевых ИПП $H_{1}$ и $H_{2}$ таких, что $H_{1} \cap H_{2}=\{0\}$ (здесь $H_{1}+H_{2}$ - замыкание алгебраической суммы подпространств). Каждое неприводимое ИПП задается комплексным числом $\mu \in \mathbb{C}_{+}$. При $\mu \neq i(2 d+2 n-1), d \in \mathbb{Z}_{+}$, все спектры $\sigma(\lambda), \lambda \in \Lambda_{0}$, должны состоять из одного числа $\mu$ с кратностью 1 . Соответствующее ИПП (обозначим его через $\mathscr{F}(\mu)$ ) состоит из всех функций $f \in \mathscr{F} \cap C^{\infty}$, удовлетворяющих дифференциальному уравнению

$$
\left(\tilde{\Delta}+\mu^{2}+\rho^{2}\right) f=0
$$


т.е. это ИПП является собственным подпространством оператора Лапласа-Бельтрами. Из того, что $\tilde{\Delta}$ - эллиптический оператор, легко получить, что это подпространство замкнуто в $\mathscr{F}$. Отметим, что неприводимость в $\mathscr{E}$ подпространства, состоящего из всех решений уравнения (1.4) при $\mu \neq i(2 d+2 n-1)$, и аналогичные результаты для других симметрических пространств ранга 1 установлены ранее С. Хелгасоном в [14].

Если $\mu=i(2 d+2 n-1)$ и $d \geqslant 1$, то спектры $\sigma(\lambda), \lambda=(k, k, l, 0, \ldots, 0) \in \Lambda_{0}$, должны состоять из числа $\mu$ с кратностью 1 при $l \leqslant d$ и $k+l+1 \leqslant d$ и должны быть пустыми при остальных $\lambda$. Число весов $\lambda \in \Lambda_{0}$, удовлетворяюших условиям $l \leqslant d$ и $k+l+1 \leqslant d$, конечно, поэтому соответствующее неприводимое ИПП конечномерно.

При $\mu=i(2 d+2 n-1)$ и $d=0$ (т.е. $\mu=i(2 n-1))$ спектры $\sigma(\lambda), \lambda=$ $(k, k, l, 0, \ldots, 0)$, должны состоять из числа $\mu$ с кратностью 1 при $l=0$ и любом $k \in \mathbb{Z}_{+}$и должны быть пустыми при $l \geqslant 1$. Соответствующее неприводимое ИПП будет бесконечномерным, но не совпадет с $\mathscr{F}(\mu)$. Отметим, что подобных неприводимых ИПП не существует на симметрических пространствах $\mathrm{SO}_{0}(n, 1) / \mathrm{SO}(n)$ и $\mathrm{SU}(1, n) / U(n)$, так как там любое ИПП либо является собственным подпространством оператора Лапласа-Бельтрами, либо конечномерно (см. [1]).

Подпространство $H$ неразложимо тогда и только тогда, когда все спектры $\sigma(\lambda)$ состоят из одного числа $\mu$ (не зависящего от $\lambda$ ) с некоторой кратностью. При $\mu \neq i(2 d+2 n-1), d \in \mathbb{Z}_{+}$, эта кратность должна быть одинакова при любых $\lambda \in \Lambda_{0}$, а при $\mu=i(2 d+2 n-1)$ кратность может меняться в зависимости от $\lambda$ так, чтобы выполнялись условия теоремы II. Если все спектры $\sigma(\lambda)$ одинаковы и состоят из числа $\mu$ с кратностью $r$, то соответствующее неразложимое ИПП состоит из всех функций $f \in \mathscr{F} \cap C^{\infty}$, удовлетворяющих дифференциальному уравнению

$$
\left(\tilde{\Delta}+\mu^{2}+\rho^{2}\right)^{r} f=0 .
$$

В общем случае произвольное ИПП является замыканием конечной или счетной прямой суммы неразложимых ИПП.

По терминологии Беренстейна [2] будем говорить, что ИПП $H \subseteq \mathscr{F}$ доnускает спектральный синтез, если $H$ совпадает с наименьшим ИПП, содержашим ячейку $H^{(0)}$, где $0=(0, \ldots, 0)$ - старший вес тривиального одномерного представления группы $K$ (в этом случае $H$ совпадает также с наименьшим ИПП, содержашим сферические функции $\Phi_{\mu}(x)$ и их производные $\partial_{\mu}^{k} \Phi_{\mu}(x), k=0,1, \ldots, r-1$, где $\mu$ пробегает спектр $\sigma(0), r$ - кратность числа $\mu)$. Из теоремы II сразу следует, что ИПП $H$ допускает спектральный синтез тогда и только тогда, когда спектры $\sigma(\lambda)$ удовлетворяют условию: спектр $\sigma\left(\lambda+\delta_{\nu}\right), \nu=1,2$, получается из $\sigma(\lambda)$ уменьшением кратности числа $\mu_{\nu}$ на 1 (если эта кратность равна 0 , то предполагается, что $\left.\sigma\left(\lambda+\delta_{\nu}\right)=\sigma(\lambda)\right)$.

В частности, получаем теорему Ваврынчика [4]: если спектр $\sigma(0)$ не содержит чисел $\mu=i(2 d+2 n-1), d \in \mathbb{Z}_{+}$, то подпространство $H$ допускает спектральньй синтез (в [4] рассматривалось пространство $\mathscr{F}=\mathscr{E}$ ).

Результаты настояшей работы частично анонсированы в [15]. 


\section{§2. Переход к пространствам $\mathscr{F}(\lambda)$}

Пусть $M=G / K$ - произвольное риманово симметрическое пространство некомпактного типа, $G$ - связная полупростая группа Ли с конечным центром, $K-$ максимальная компактная подгрупша в $G$. Пусть $\mathfrak{g}_{0}$ и $\mathfrak{k}_{0}$ - алгебры Ли групп $G$ и $K$ соответственно. Если $\pi$ - произвольное представление группы $G$ в полном ЛВП $\mathscr{F}$, то вектор $\xi \in \mathscr{F}$ называется гладким (аналитическим), когда отображение $g \rightarrow \pi(g) \xi$ бесконечно дифференцируемо (соответственно аналитическое). Вектор $\xi$ называется $K$-финитным, если линейная оболочка векторов $\pi(u) \xi$ при $u \in K$ конечномерна. Пусть $\mathscr{F}_{\sigma}$ - множество всех гладких $K$-финитных векторов, а $\mathscr{F} \#-$ множество аналитических $K$-финитных векторов пространства $\mathscr{F}$. Линейные подпространства $\mathscr{F}_{\sigma}$ и $\mathscr{F}_{\#}$ инвариантны относительно индуцированного представления алгебры Ли $\mathfrak{g}_{0}$.

Вернемся к случаю, когда $\mathscr{F}$ - функциональное пространство типа 1 или типа 2 , $\pi$ - квазирегулярное представление групшы $G$. Для любого ИПП $H \subset \mathscr{F}$ пусть $H_{\sigma}=H \cap \mathscr{F}_{\sigma}, H_{\#}=H \cap \mathscr{F}_{\#}$. Обозначим через $\Lambda$ множество старших весов неприводимых конечномерных представлений группы $K$, пусть $E^{\lambda}$ - пространство неприводимого представления со старшим весом $\lambda \in \Lambda,\langle$,$\rangle - инвариантная эр-$ митова форма в $E^{\lambda}$. Пространства $\mathscr{F}^{(\lambda)}$ определены в $\S 1$. Через $\mathscr{F}_{\sigma}^{(\lambda)}\left(\right.$ или $\mathscr{F}_{\#}^{(\lambda)}$ ) обозначим множество всех функций $F(x) \in \mathscr{F}^{(\lambda)}$, удовлетворяюших условию

$$
f_{\xi}(x)=\langle F(x), \xi\rangle \in \mathscr{F}_{\sigma} \quad\left(\text { соответственно } f_{\xi}(x) \in \mathscr{F}_{\#}\right) \quad \forall \xi \in E^{\lambda} .
$$

Если $H-$ ИПП в $\mathscr{F}$, то ячейки $H^{(\lambda)}$ определены в $\S 1$. Пусть $H_{\sigma}^{(\lambda)}=H^{(\lambda)} \cap \mathscr{F}_{\sigma}^{(\lambda)}$, $H_{\#}^{(\lambda)}=H^{(\lambda)} \cap \mathscr{F}_{\#}^{(\lambda)}$. Пусть $\mathfrak{g}_{0}=\mathfrak{k}_{0}+\mathfrak{p}_{0}-$ разложение Картана алгебры Ли $\mathfrak{g}_{0} ; \mathfrak{g}, \mathfrak{k}$ и $\mathfrak{p}-$ комплексификации пространств $\mathfrak{g}_{0}, \mathfrak{k}_{0}$ и $\mathfrak{p}_{0}$ соответственно. Так как $[\mathfrak{k}, \mathfrak{p}] \subseteq \mathfrak{p}$, то $\mathfrak{p}$ является $\mathfrak{k}$-модулем. Пусть $p_{1}, \ldots, p_{m}$ - базис в $\mathfrak{p}$, причем пусть все $p_{j} \in \mathfrak{p}_{0}$; пусть $\mathfrak{p}^{*}$ - двойственньй $\mathfrak{p}$-модуль, а $p_{1}^{*}, \ldots, p_{m}^{*}$ - двойственный базис в $\mathfrak{p}^{*}$.

Для $\lambda, \mu \in \Lambda$ обозначим через $\operatorname{Hom}\left(E^{\mu}, E^{\lambda}\right)$ множество линейных операторов из $E^{\mu}$ в $E^{\lambda}$. Пространство $\operatorname{Hom}\left(E^{\mu}, E^{\mu}\right)$ является k-модулем (т.е. $(k A) \xi=k(A \xi)-$ $A(k \xi)$ для любых $\left.\xi \in E^{\mu}, k \in \mathfrak{k}\right)$. Пусть

$$
\varphi: \mathfrak{p}^{*} \rightarrow \operatorname{Hom}\left(E^{\mu}, E^{\lambda}\right)
$$

- произвольный гомоморфизм $\mathfrak{k}$-модулей.

Положим $\alpha_{j}=\varphi\left(p_{j}\right) \in \operatorname{Hom}\left(E^{\mu}, E^{\lambda}\right)$, и пусть $\alpha_{j}^{*}=\varphi\left(p_{j}^{*}\right) \in \operatorname{Hom}\left(E^{\lambda}, E^{\mu}\right)-$ сопряженный оператор (т.е. $\left\langle\alpha_{j} \xi, \eta\right\rangle=\left\langle\xi, \alpha_{j}^{*} \eta\right\rangle \forall \xi \in E^{\mu}, \forall \eta \in E^{\lambda}$ ).

Для функции $F(x) \in \mathscr{F}_{\sigma}^{(\lambda)}$ определим действие оператора $L(\varphi)$ формулой

$$
(L(\varphi) F)(x)=-\sum_{j=1}^{m} \alpha_{j}^{*}\left(\left(p_{j} F\right)(x)\right)
$$

где $p_{j} F$ - действие элемента из алгебры Ли на функцию, определенное формулой (1.2). 
Известно (см. $[1, \S 3])$, что оператор $L(\varphi)$ переводит пространство $\mathscr{F}_{\sigma}^{(\lambda)}$ в $\mathscr{F}_{\sigma}^{(\mu)}$ и пространство $\mathscr{F}_{\#}^{(\lambda)}$ в $\mathscr{F}_{\#}^{(\mu)}$. Более того, если $H^{(\lambda)}$ и $H^{(\mu)}-$ ячейки одного ИПП, то $L(\varphi)\left(H_{\sigma}^{(\lambda)}\right) \subseteq H_{\sigma}^{(\mu)}$ и $L(\varphi)\left(H_{\#}^{(\lambda)}\right) \subseteq H_{\#}^{(\mu)}$. Операторы $L(\varphi)$ служат основными инструментами для доказательства теорем I и II. Обозначим множество операторов $L(\varphi)$, построенных по всевозможным гомоморфизмам $(2.2)$, через $P_{0}(\lambda, \mu)$.

При доказательствах будет часто использоваться следующая лемма.

Лемма 2.1. Пусть $\mathfrak{k}$ - произвольная алгебра Ли, векторы $X_{\alpha}, X_{-\alpha}, H_{\alpha}$ принадлежат ḱ, причем

$$
\left[H_{\alpha}, X_{\alpha}\right]=2 X_{\alpha}, \quad\left[H_{\alpha}, X_{-\alpha}\right]=-2 X_{-\alpha}, \quad\left[X_{\alpha}, X_{-\alpha}\right]=H_{\alpha}
$$

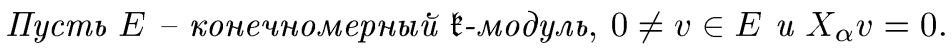

Тогда:

1) $H_{\alpha} v=d v$, где $d \in \mathbb{Z}_{+}$;

2) векторы $X_{-\alpha}^{r} v \neq 0$ при $r=0,1, \ldots, d u X_{-\alpha}^{d+1} v=0$;

3) $X_{\alpha}\left(X_{-\alpha}^{r} v\right)=r(d-r+1) X_{-\alpha}^{r-1} v$.

Доказательство сразу следует из теории представлений алгебры Ли $\mathrm{sl}(2, \mathbb{C})$ (см. [13, гл. VIII, §1]).

Далее, пусть $M=\mathrm{Sp}(1, n) / \mathrm{Sp}(1) \times \mathrm{Sp}(n)$. Тогда алгебра Ли $\mathfrak{g}_{0}=\mathrm{sp}(1, n)$. Ее комплексификация $\mathfrak{g}=\operatorname{sp}(n+1, \mathbb{C})=\operatorname{sp}(n+1)$ состоит из матриц

$$
\left(\begin{array}{cc}
A & B \\
C & -A^{t}
\end{array}\right)
$$

где $A, B, C$ - комплексные матрицы размера $(n+1) \times(n+1)$, причем $B^{t}=B$ и $C^{t}=C$. Будем считать, что индексы в матрицах $A, B, C$ из (2.4) меняются от 0 до $n$. Подалгебра Ли k и подпространство $\mathfrak{p}$ состоят соответственно из матриц

$$
k=\left(\begin{array}{cccc}
a & 0 & b & 0 \\
0 & A_{1} & 0 & B_{1} \\
c & 0 & -a & 0 \\
0 & C_{1} & 0 & -A_{1}^{t}
\end{array}\right), \quad p=\left(\begin{array}{cccc}
0 & z_{1} & 0 & z_{2} \\
-z_{4}^{t} & 0 & z_{2}^{t} & 0 \\
0 & z_{3} & 0 & z_{4} \\
z_{3}^{t} & 0 & -z_{1}^{t} & 0
\end{array}\right)
$$

где $a, b, c \in \mathbb{C}, A_{1}, B_{1}, C_{1}$ - комплексные матрицы размера $n \times n$, причем $B_{1}^{t}=B_{1}$ и $C_{1}^{t}=C_{1}, z_{1}, z_{2}, z_{3}, z_{4}$ - строки длины $n$. Подалгебра Ли ⿱ㅏㅇ состоит из пересечения $\mathfrak{k}$ с множеством косоэрмитовых матриц, подпространство $\mathfrak{p}_{0}$ равно пересечению $\mathfrak{p}$ с множеством эрмитовых матриц. При $A_{1}=B_{1}=C_{1}=0$ в k возникает подалгебра Ли, изоморфная алгебре Ли $\mathrm{sp}(1)=\mathrm{sl}(2)$, а при $a=b=c=0$ возникает подалгебра, изоморфная $\operatorname{sp}(n)$. В целом $\mathfrak{k} \simeq \operatorname{sp}(1) \times \operatorname{sp}(n)$. Подалгебра Картана $\mathfrak{h}$ в алгебре $\mathfrak{g}$ (а также и в $\mathfrak{k})$ состоит из всех диагональных матриц

$$
h=\operatorname{diag}\left(h_{0}, h_{1}, \ldots, h_{n},-h_{0},-h_{1}, \ldots,-h_{n}\right)=\gamma\left(h_{0}, h_{1}, \ldots, h_{n}\right),
$$


где $h_{j} \in \mathbb{C}$.

Линейный функционал $\varepsilon_{j} \in \mathfrak{h}^{*}(j=0,1, \ldots, n)$ определим условием $\varepsilon_{j}(h)=h_{j}$. Тогда корнями алгебры Ли $\mathfrak{g}$ будут функционалы $\left(\varepsilon_{j}+\varepsilon_{r}\right)$ при $0 \leqslant j \leqslant r \leqslant n$, $\left(\varepsilon_{j}-\varepsilon_{r}\right)$ при $0 \leqslant j \neq r \leqslant n$ и $\left(-\varepsilon_{j}-\varepsilon_{r}\right)$ при $0 \leqslant j \leqslant r \leqslant n$. Будем обозначать эти корни соответственно $\omega_{j r}, \omega_{j r^{\prime}}$ и $\omega_{j^{\prime} r^{\prime}}$ (т. е. $\omega_{j r}(h)=h_{j}+h_{r}, \omega_{j r^{\prime}}(h)=h_{j}-h_{r}$, $\left.\omega_{j^{\prime} r^{\prime}}(h)=-h_{j}-h_{r}\right)$.

Матрицы $X_{j r}, X_{j r^{\prime}}=X_{r^{\prime} j}, X_{j^{\prime} r^{\prime}} \in \mathfrak{g}=\operatorname{sp}(n+1)$ при $j, r=0,1, \ldots, n$ определим через соответствуюшие им матрищы $A, B$ и $C$ из (2.4):

для $X_{j r}$ пусть $A=C=0, B=E_{j r}+E_{r j}$ при $r \neq j$ и $B=E_{j j}$ при $r=j$;

для $X_{j^{\prime} r^{\prime}}$ пусть $A=B=0, C=E_{j r}+E_{r j}$ при $r \neq j$ и $C=E_{j j}$ при $r=j$;

для $X_{j r^{\prime}}$ и $X_{r^{\prime} j}$ пусть $B=C=0, A=E_{j r}$, где $E_{j r}$-матрица с 1 на месте $(j, r)$ и с нулями на остальных местах.

Тогда для любого $h \in \mathfrak{h}$

$$
\left[h, X_{j r}\right]=\omega_{j r}(h) X_{j r}, \quad\left[h, X_{j r^{\prime}}\right]=\omega_{j r^{\prime}}(h) X_{j r^{\prime}}, \quad\left[h, X_{j^{\prime} r^{\prime}}\right]=\omega_{j^{\prime} r^{\prime}}(h) X_{j^{\prime} r^{\prime}}
$$

т. е. векторы $X_{j r}, X_{j r^{\prime}}$ и $X_{j^{\prime} r^{\prime}}$ являются корневыми векторами для соответствуюших корней. Отметим, что

$$
X_{j r}=X_{r j}, \quad X_{j^{\prime} r^{\prime}}=X_{r^{\prime} j^{\prime}}, \quad X_{j r^{\prime}}=X_{r^{\prime} j} .
$$

Удобно также считать, что $X_{j j^{\prime}}=0$.

Определим еще матрищы

$$
H_{j r}=\gamma\left(e_{j}+e_{r}\right), \quad H_{j r^{\prime}}=H_{r^{\prime} j}=\gamma\left(e_{j}-e_{r}\right), \quad H_{j^{\prime} r^{\prime}}=\gamma\left(-e_{j}-e_{r}\right)
$$

при $j \neq r$ и

$$
H_{j j}=\gamma\left(e_{j}\right), \quad H_{j^{\prime} j^{\prime}}=\gamma\left(-e_{j}\right),
$$

где $e_{j}$ - строка длины $n+1$ с 1 на $j$-м месте и с нулями на остальных местах, $\gamma$ определено формулой (2.6). Отметим, что $H_{j^{\prime} r^{\prime}}=-H_{j r}$ и $H_{j r^{\prime}}=-H_{j^{\prime} r}$.

Если $\alpha$ - один из корней $\omega_{j r}, \omega_{j r^{\prime}}, \omega_{j^{\prime} r^{\prime}}$, то через $X_{\alpha}$ и $H_{\alpha}$ будем обозначать соответственно матрицы $X_{j r}, X_{j r^{\prime}}, X_{j^{\prime} r^{\prime}}$ и $H_{j r}, H_{j r^{\prime}}, H_{j^{\prime} r^{\prime}}$. Легко видеть, что при этом $\left[X_{\alpha}, X_{-\alpha}\right]=H_{\alpha}$ и $\alpha\left(H_{\alpha}\right)=2$. Напомним, что если $\alpha, \beta$-произвольные корни полупростой алгебры Ли, причем $\alpha+\beta \neq 0$ и $\alpha+\beta$ не является корнем, $X_{\alpha}$ и $X_{\beta}$ - корневые векторы, то $\left[X_{\alpha}, X_{\beta}\right]=0$. В нашем случае ненулевыми будут только следуюшие коммутаторы:

$$
\begin{aligned}
{\left[X_{s j^{\prime}}, X_{j r}\right] } & =X_{s r}, \quad\left[X_{s^{\prime} j^{\prime}}, X_{j r^{\prime}}\right]=X_{s^{\prime} r^{\prime}} \\
{\left[X_{s j}, X_{j^{\prime} r^{\prime}}\right] } & =X_{s r^{\prime}}, \quad\left[X_{s j^{\prime}}, X_{j r^{\prime}}\right]=X_{s r^{\prime}}
\end{aligned}
$$

при $j \neq r$ и

$$
\begin{array}{rlrl}
{\left[X_{s j^{\prime}}, X_{j s}\right]} & =2 X_{s s}, & {\left[X_{s^{\prime} j^{\prime}}, X_{j s^{\prime}}\right]} & =2 X_{s^{\prime} s^{\prime}}, \\
{\left[X_{s j}, X_{j^{\prime} s^{\prime}}\right]} & =2 H_{s j}, \quad\left[X_{s j^{\prime}}, X_{j s^{\prime}}\right]=2 H_{s j^{\prime}},
\end{array}
$$


где $j, r, s=0,1, \ldots, n$, и коммутаторы, получающиеся из них изменением порядка матриц и порядка индексов по формулам (2.7).

Корнями подалгебры k являются функционалы $\omega_{00}, \omega_{0^{\prime} 0^{\prime}}$ и функционалы $\omega_{j r}$, $\omega_{j r^{\prime}}, \omega_{j^{\prime} r^{\prime}}$ при $1 \leqslant j, r \leqslant n$. Из них положительными корнями будут $\omega_{00}, \omega_{j r}$ при $0 \leqslant j, r \leqslant n$ и $\omega_{j r^{\prime}}$ при $j<r$. Простыми корнями являются

$$
\alpha_{0}=\omega_{00}, \quad \alpha_{1}=\omega_{12^{\prime}}, \quad \alpha_{2}=\omega_{23^{\prime}}, \quad \ldots, \quad \alpha_{n-1}=\omega_{n-1, n^{\prime}}, \quad \alpha_{n}=\omega_{n n} .
$$

Любой линейный функционал $\lambda \in \mathfrak{h}^{*}$ будем часто отождествлять с набором чисел $\lambda=\left(\lambda_{0}, \lambda_{1}, \ldots, \lambda_{n}\right)$, где $\lambda_{j}=\lambda\left(H_{\alpha_{j}}\right)$. При этом $\lambda$ является старшим весом не-

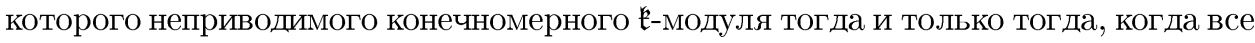
числа $\lambda_{j} \in \mathbb{Z}_{+}$, т.е. вес $\lambda$ доминантный.

Так как $[\mathfrak{k}, \mathfrak{p}] \subseteq \mathfrak{p}$, то $\mathfrak{p}$ является $\mathfrak{k}$-модулем. Всевозможные веса этого модуля следуюшие:

$$
\pm \omega_{01}, \quad \pm \omega_{02}, \ldots, \quad \pm \omega_{0 n} ; \quad \pm \omega_{01^{\prime}}, \quad \pm \omega_{02^{\prime}}, \ldots, \quad \pm \omega_{0 n^{\prime}}
$$

Модуль р неприводим, и его старший вес равен $\omega_{01}$. Пусть $E^{\lambda}$ и $E^{\mu}-$ неприво-

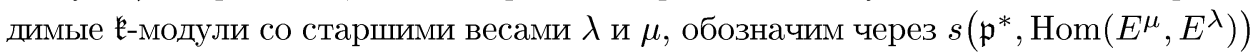
множество гомоморфизмов $\mathfrak{k}$-модулей (сплетающих операторов) из двойственного модуля $\mathfrak{p}^{*}$ в $\operatorname{Hom}\left(E^{\mu}, E^{\lambda}\right)$. Заметим, что следующие множества сплетающих операторов изоморфны как векторные пространства:

$$
s\left(\mathfrak{p}^{*}, \operatorname{Hom}\left(E^{\mu}, E^{\lambda}\right)\right) \simeq s\left(E^{\mu}, E^{\lambda} \otimes \mathfrak{p}\right) .
$$

Из результатов работы [16] сразу следует, что

$$
E^{\lambda} \otimes \mathfrak{p}=\oplus E^{\lambda+\omega}
$$

где $\omega$ пробегает все веса (2.11). Если $\lambda+\omega$ не является доминантным весом, то соответствуюшее слагаемое в (2.13) заменяется нулем.

Из соотношений (2.12) и (2.13) следует, что векторное пространство $s\left(\mathfrak{p}^{*}, \operatorname{Hom}\left(E^{\mu}, E^{\lambda}\right)\right)$ ненулевое тогда и только тогда, когда $\mu=\lambda+\omega$, где $\omega$ - один из весов (2.11), и при этом размерность этого пространства равна 1. В результате доказано следующее

ПРЕДЛОЖЕНИЕ 2.2. $\operatorname{dim} P_{0}(\lambda, \mu) \leqslant 1$ для любъх $\lambda, \mu \in \Lambda$, при әтом необходимым условием того, ито $P_{0}(\lambda, \mu) \neq\{0\}$, является $\mu=\lambda+\omega$, где $\omega-$ один из весов (2.11).

Пусть

$$
I_{0}=X_{01^{\prime}}+X_{10^{\prime}}, \quad \alpha(t)=\exp \left(t I_{0}\right) .
$$

Тогда $I_{0} \in \mathfrak{p}_{0}$, а $\alpha(t)$ - однопараметрическая подгруппа в группе $G$. Подалгебру Ли $\mathfrak{k}_{1}$ в $\mathfrak{k}$ определим условием

$$
\mathfrak{k}_{1}=\left\{k \in \mathfrak{k}:\left[k, I_{0}\right]=0\right\},
$$


и пусть $K_{1}$ - связная подгруппа Ли в группе $K$, соответствуюшая подалгебре Ли $\mathfrak{k}_{1}$. Тогда $u \alpha(t)=\alpha(t) u$ для $u \in K$. Как векторное пространство подалгебра $\mathfrak{k}_{1}$ порождается матрицами $H_{00}+H_{11}, X_{00}-X_{11}, X_{0^{\prime} 0^{\prime}}-X_{1^{\prime} 1^{\prime}}$ и матрицами $H_{j j}$, $X_{j r}, X_{j r^{\prime}}, X_{j^{\prime} r^{\prime}}$ при $2 \leqslant j, r \leqslant n$.

Пусть $\Lambda_{0}-$ множество старших весов вида $\lambda=(k, k, l, 0, \ldots, 0)$, т.е. $\lambda_{0}=\lambda_{1}=k$, $\lambda_{2}=l$ и $\lambda_{3}=\cdots=\lambda_{n}=0$.

ПРЕДЛОЖЕНИЕ 2.3. Пусть $F(x)$ - ненулевая функиия на $M$, принимающая значения в $E^{\lambda}$ и удовлетворяющая условию

$$
F(u x)=T^{\lambda}(u) F(x) \quad \forall u \in K
$$

Тогда $\lambda \in \Lambda_{0}$.

ДокАЗАТЕЛЬСТво. Пусть $o=\mathrm{e} K$ - фиксированная точка пространства $M=G / K, \mathrm{e}-$ единичный элемент группы $G$. Любая точка пространства $M$ имеет вид $x=g o$, где $g \in G$. Известно (см., например, [8]), что любой элемент из групшы $G$ можно представить в виде $g=u_{1} \alpha(t) u_{2}$, где $u_{1}, u_{2} \in K$. Тогда

$$
F(x)=F\left(u_{1} \alpha(t) u_{2} o\right)=T^{\lambda}\left(u_{1}\right) F(\alpha(t) o)
$$

Следовательно, функция $F(\alpha(t) o)$ не равна тождественно нулю. Если вектор $\xi=F(\alpha(t) 0) \neq 0, u \in K_{1}$, то

$$
T^{\lambda}(u) \xi=F(u \alpha(t) o)=F(\alpha(t) u o)=F(\alpha(t) o)=\xi .
$$

Следовательно, $k \xi=0 \forall k \in \mathfrak{k}_{1}$. В результате доказано, что в пространстве $E^{\lambda}$ должен существовать вектор $\xi \neq 0$ такой, что $\mathfrak{k}_{1} \xi=0$.

Подберем наибольшее число $k \in \mathbb{Z}_{+}$такое, что $X_{00}^{k} \xi \neq 0$ (тогда $X_{00}^{k+1} \xi=0$ ). Далее возьмем наибольшее $l \in \mathbb{Z}_{+}$такое, что $v=X_{12}^{l}\left(X_{00}^{k} \xi\right) \neq 0$. Проверим, что $v$ является старшим вектором пространства $E^{\lambda}$. Для этого достаточно проверить, что $X_{\alpha_{j}} v=0$ для $j=0,1, \ldots, n$.

При $j \geqslant 2$ векторы $X_{\alpha_{j}} \in \mathfrak{k}_{1}$ коммутируют с $X_{12}$ и $X_{00}$, поэтому $X_{\alpha_{j}} v=$ $X_{12}^{l} X_{00}^{k} X_{\alpha_{j}} \xi=0$. Далее, $X_{\alpha_{0}} v=X_{00} v=X_{12}^{l} X_{00}^{k+1} \xi=0$.

Заметим, что $X_{\alpha_{1}}=X_{12^{\prime}}=\left[X_{12}, X_{2^{\prime} 2^{\prime}}\right]$ и $X_{2^{\prime} 2^{\prime}} \in \mathfrak{k}_{1}$. Кроме того, $X_{00}-X_{11} \in \mathfrak{k}_{1}$, следовательно, $X_{00} \xi=X_{11} \xi$ и $X_{11} X_{00}^{k} \xi=X_{00}^{k+1} \xi=0$. Тогда

$$
\begin{aligned}
X_{\alpha_{1}} v & =\left(X_{12} X_{2^{\prime} 2^{\prime}}-X_{2^{\prime} 2^{\prime}} X_{12}\right) v=X_{12} X_{2^{\prime} 2^{\prime}} X_{12}^{l} X_{00}^{k} \xi \\
& =X_{12}\left(X_{2^{\prime} 2^{\prime}} X_{12}^{l}-X_{12}^{l} X_{2^{\prime} 2^{\prime}}\right) X_{00}^{k} \xi=-l X_{12^{\prime}} X_{12}^{l} X_{00}^{k} v=-l X_{\alpha_{1}} v .
\end{aligned}
$$

Следовательно, $(l+1) X_{\alpha_{1}} v=0$ и $X_{\alpha_{1}} v=0$. Тем самым $v$ будет старшим вектором, в частности весовым вектором веса $\lambda$.

Так как $v$-весовой вектор веса $\lambda$, то $H_{\alpha_{j}} v=\lambda_{j} v$. С другой стороны, заметим, что $H_{\alpha_{j}} \in \mathfrak{k}_{1}$ при $j \geqslant 2,\left[H_{\alpha_{j}}, X_{12}\right]=0$ при $j \geqslant 3$ и $\left[H_{\alpha_{2}}, X_{12}\right]=X_{12}$. Тогда 
$\lambda_{j} v=H_{\alpha_{j}} X_{12}^{l} X_{00}^{k} \xi=X_{12}^{l} X_{00}^{k} H_{\alpha_{j}} \xi=0$ для $j \geqslant 3, \lambda_{2} v=H_{\alpha_{2}} X_{12}^{l} X_{00}^{k} \xi=$ $\left(H_{\alpha_{2}} X_{12}^{l}-X_{12}^{l} H_{\alpha_{2}}\right) X_{00}^{k} \xi=l X_{12}^{l} X_{00}^{k} \xi=l v$.

Заметим, что $H_{\alpha_{0}}=\left[X_{00}, X_{0^{\prime} 0^{\prime}}\right]$. Тогда

$$
\begin{aligned}
\lambda_{0} v= & \left(X_{00} X_{0^{\prime} 0^{\prime}}-X_{0^{\prime} 0^{\prime}} X_{00}\right) X_{12}^{l} X_{00}^{k} \xi=X_{12}^{l} X_{00} X_{0^{\prime} 0^{\prime}} X_{00}^{k} \xi \\
= & -X_{12}^{l} X_{00}\left(H_{\alpha_{0}} X_{00}^{k-1}+X_{00} H_{\alpha_{0}} X_{00}^{k-2}+\cdots+X_{00}^{k-1} H_{\alpha_{0}}\right) \xi \\
& +X_{12}^{l} X_{00}^{k+1} X_{0^{\prime} 0^{\prime}} \xi .
\end{aligned}
$$

Последнее слагаемое в (2.16) равно нулю, так как $X_{0^{\prime} 0^{\prime}}-X_{1^{\prime} 1^{\prime}} \in \mathfrak{k}_{1}$ и $X_{00}^{k+1} X_{0^{\prime} 0^{\prime}} \xi=$ $X_{00}^{k+1} X_{1^{\prime} 1^{\prime}} \xi=X_{1^{\prime} 1^{\prime}} X_{00}^{k+1} \xi=0$. Из равенства $\left[H_{\alpha_{0}}, X_{00}\right]=2 X_{00}$ следует, что $X_{00}^{r} H_{\alpha_{0}}=H_{\alpha_{0}} X_{00}^{r}-2 r X_{00}^{r}$, и из (2.16) получаем, что $\lambda_{0} v=-k H_{\alpha_{0}} v+$ $(2+4+\cdots+2 k) v$. Так как $H_{\alpha_{0}} v=\lambda_{0} v$, то $(k+1) \lambda_{0} v=k(k+1) v$ и, следовательно, $\lambda_{0}=k$.

Пусть $H=H_{\alpha_{0}}+H_{\alpha_{1}} \in \mathfrak{k}_{1}$. Легко видеть, что $\left[H, X_{12}\right]=0$ и $\left[H, X_{00}\right]=2 X_{00}$, откуда следует, что $\left(H X_{12}^{l} X_{00}^{k}-X_{12}^{l} X_{00}^{k} H\right)=2 k X_{12}^{l} X_{00}^{k}$. Так как $H \in \mathfrak{k}_{1}$, то $H \xi=0$ и $\left(\lambda_{0}+\lambda_{1}\right) v=H v=\left(H X_{12}^{l} X_{00}^{k}-X_{12}^{l} X_{00}^{k} H\right) \xi=2 k v$. Следовательно, и $\lambda_{1}=k$. Окончательно $\lambda_{0}=\lambda_{1}=k, \lambda_{2}=l, \lambda_{3}=\cdots=\lambda_{n}=0$.

Операцию эрмитова сопряжения на алгебре $\mathfrak{k}$ продолжим до эрмитовой инволюции $a \rightarrow a^{*}$ универсальной обертывающей алгебры $U(\mathfrak{k})$. При этом $(a b)^{*}=b^{*} a^{*}$ для любых $a, b \in U(\mathfrak{k}), X_{\alpha}^{*}=X_{-\alpha}$ и $H_{\alpha}^{*}=H_{\alpha}$ для любого корня $\alpha$. Если $\langle\xi, \eta\rangle-$ инвариантная эрмитова форма на пространстве $E^{\lambda}$, то

$$
\langle a \xi, \eta\rangle=\left\langle\xi, a^{*} \eta\right\rangle \quad \forall a \in U(\mathfrak{k}) .
$$

Для любого k-модуля $E^{\lambda}$ пусть

$$
E_{0}^{\lambda}=\left\{\xi \in E^{\lambda}: \mathfrak{k}_{1} \xi=0\right\}
$$

ПРЕДЛОЖЕНИЕ 2.4. Пусть $E^{\lambda}$ - неприводимый $\mathfrak{k}$-модуль со стариим весом $\lambda$. Подпространство $E_{0}^{\lambda} \neq\{0\}$ тогда и только тогда, когда $\lambda \in \Lambda_{0}$. Eсли $\lambda \in \Lambda_{0}$, mo $\operatorname{dim} E_{0}^{\lambda}=1$.

ДокАЗАТЕЛЬСТво. А) В ходе доказательства предложения 2.3 уже было установлено, что из $E_{0}^{\lambda} \neq\{0\}$ следует $\lambda \in \Lambda_{0}$. Пусть $\lambda=(k, k, l, 0, \ldots, 0)$. Проверим, что $\operatorname{dim} E_{0}^{\lambda} \leqslant 1$. Если $0 \neq \xi \in E_{0}^{\lambda}$, то при доказательстве предложения 2.3 было показано, что вектор $v=X_{12}^{l} X_{00}^{k} \xi \neq 0$ является старшим вектором пространства $E^{\lambda}$. Следовательно, отображение $\xi \rightarrow X_{12}^{l} X_{00}^{k} \xi$ инъективно и переводит $E_{0}^{\lambda}$ в одномерное пространство векторов старшего веса $\lambda$. Поэтому $\operatorname{dim} E_{0}^{\lambda} \leqslant 1$.

Остается проверить, что в $E_{0}^{\lambda}$ есть ненулевые векторы. Введем следующий элемент из $U(\mathfrak{k})$ :

$$
\mathscr{D}=X_{1^{\prime} 2^{\prime}} H_{23^{\prime}}+\sum_{d=3}^{n} X_{1^{\prime} d^{\prime}} X_{2^{\prime} d}-\sum_{d=2}^{n} X_{1^{\prime} d} X_{2^{\prime} d^{\prime}} .
$$


Пусть $v_{0}-$ старший вектор пространства $E^{\lambda}$,

$$
\begin{gathered}
w_{0}=\mathscr{D}^{l} v_{0}, \\
\xi_{0}=\sum_{r=0}^{k} X_{0^{\prime} 0^{\prime}}^{r} X_{1^{\prime} 1^{\prime}}^{k-r} w_{0} .
\end{gathered}
$$

Далее будет показано, что $\xi_{0} \neq 0$ и $\xi_{0} \in E_{0}^{\lambda}$; это и завершит доказательство.

Пусть $\mu \in \mathfrak{h}^{*}, a \in U(\mathfrak{k})$. Будем говорить, что $a$ - элемент веса $\mu$, если

$$
[h, a]=h a-a h=\mu(h) a \quad \forall h \in \mathfrak{h} .
$$

Как и раньше, функционал $\mu$ отождествляется со строкой $\mu=\left(\mu_{0}, \mu_{1}, \ldots, \mu_{n}\right)$, где $\mu_{j}=\mu\left(H_{\alpha_{j}}\right)$. Тогда $\mathscr{D}$ - элемент веса $\omega_{1^{\prime} 2^{\prime}}=(0,0,-1,0, \ldots, 0)$.

Б) Пусть $v_{r}=\mathscr{D}^{r} v_{0}$. Индукцией по $r$ проверим, что

$$
X_{\alpha_{j}} v_{r}=0 \text { при } j=0 \text { и } j=2,3, \ldots, n,
$$

или, что эквивалентно,

$$
X_{00} v_{r}=0 \text { и } X_{j s^{\prime}} v_{r}=X_{j s} v_{r}=0 \text { при } 2 \leqslant j \leqslant s \leqslant n .
$$

При $r=0$ это верно, так как $v_{0}$ - старший вектор. Пусть $(2.22)$ верно для $r-1$. Легко видеть, что

$$
\begin{gathered}
{\left[X_{\alpha_{j}}, \mathscr{D}\right]=0 \text { при } j=0 \text { или } j \geqslant 4,} \\
{\left[X_{\alpha_{3}}, \mathscr{D}\right]=X_{1^{\prime} 2^{\prime}} X_{\alpha_{3}},} \\
{\left[X_{\alpha_{2}}, \mathscr{D}\right]=-2 X_{1^{\prime} 2^{\prime}} X_{\alpha_{2}}-\sum_{d=4}^{n} X_{1^{\prime} d^{\prime}} X_{3^{\prime} d}} \\
+2 X_{1^{\prime} 3} X_{3^{\prime} 3^{\prime}}+\sum_{d=4}^{n} X_{1^{\prime} d} X_{3^{\prime} d^{\prime}}
\end{gathered}
$$

Из (2.24) сразу следует, что $X_{\alpha_{j}} v_{r}=0$ при $j=0$ или $j \geqslant 4$. Из (2.25) и предположения индукции получаем, что

$$
X_{\alpha_{3}} v_{r}=X_{\alpha_{3}} \mathscr{D} v_{r-1}=\left(\mathscr{D}+X_{1^{\prime} 2^{\prime}}\right) X_{\alpha_{3}} v_{r-1}=0 .
$$

Вектор $v_{r-1}$ весовой веса $\lambda-(r-1) \omega_{12}=(k, k, l-r+1,0, \ldots, 0)$. Поэтому $H_{j s} v_{r-1}=0$ и $H_{j s^{\prime}} v_{r-1}=0$ при $3 \leqslant j, s \leqslant n$. По предположению индукции $X_{j s} v_{r-1}=0$ и $X_{j s^{\prime}} v_{r-1}=0$ при $3 \leqslant j \leqslant s \leqslant n$. Тогда из леммы 2.1 следует 
(если взять $\alpha=\omega_{j s}$ или $\alpha=\omega_{j s^{\prime}}$ и $d=0$ ), что $X_{j^{\prime} s^{\prime}} v_{r-1}=0$ и $X_{j^{\prime} s} v_{r-1}=0$ при $3 \leqslant j \leqslant s \leqslant n$. Используя $(2.26)$, получаем

$$
X_{\alpha_{2}} v_{r}=X_{\alpha_{2}} \mathscr{D} v_{r-1}=\mathscr{D} X_{\alpha_{2}} v_{r-1}+\left[X_{\alpha_{2}}, \mathscr{D}\right] v_{r-1}=0
$$

В) Проверим, что $\xi_{0} \in E_{0}^{\lambda}$.

Подалгебра k⿴囗十 порождается (как алгебра Ли) векторами $X_{00}-X_{11}, X_{0^{\prime} 0^{\prime}}-X_{1^{\prime} 1^{\prime}}$ и $X_{\alpha_{j}}, X_{-\alpha_{j}}$ при $j=2,3, \ldots, n$. Поэтому достаточно показать, что $\xi_{0}$ аннулируется этими векторами. Так как вектор $w_{0}=\mathscr{D}^{l} v_{0}$ является вектором веса $(k, k, 0, \ldots, 0)$, то $H_{\alpha_{j}} w_{0}=0$ при $j \geqslant 2$. Кроме того, $X_{\alpha_{j}} w_{0}=0$ и, следовательно, по лемме $2.1 X_{-\alpha_{j}} w_{0}=0$ при $j \geqslant 2$. Отметим также, что тогда и $X_{2^{\prime} 2^{\prime}} w_{0}=0$. Так как $X_{\alpha_{j}}$ и $X_{-\alpha_{j}}$ при $j \geqslant 2$ коммутируют с $X_{0^{\prime} 0^{\prime}}$ и $X_{1^{\prime} 1^{\prime}}$, то $X_{\alpha_{j}} \xi_{0}=0, X_{-\alpha_{j}} \xi_{0}=0$.

Из п.Б) следует, что $X_{00} w_{0}=0$. Проверим, что $X_{11} w_{0}=0$. Предположим противное, т.е. что $X_{11} w_{0} \neq 0$. Подберем наибольшее $d \in \mathbb{Z}_{+}$, для которого $\widetilde{w}=$ $X_{11}^{d} w_{0} \neq 0$. Затем подберем наибольшее $m \in \mathbb{Z}_{+}$такое, что $X_{12}^{m} \widetilde{w} \neq 0$. Дословно повторяя рассуждения из доказательства предложения 2.3 , получим, что $X_{12}^{m} \widetilde{w}$ является старшим вектором в $E^{\lambda}$. Но $X_{11}$ - вектор веса $(0,2,0, \ldots, 0)$ и $X_{12}-$ вектор веса $(0,0,1,0, \ldots, 0)$; тогда $X_{12}^{m} \widetilde{w}$ - вектор веса $\mu=(k, k+2 d, m, 0, \ldots, 0)$, а старший вес $\lambda=(k, k, l, 0, \ldots, 0) \neq \mu$. Следовательно, $X_{11} w_{0}=0$.

Заметим, что $H_{00} w_{0}=H_{11} w_{0}=k w_{0}$, поэтому из леммы 2.1 следует, что $X_{0^{\prime} 0^{\prime}}^{k+1} w_{0}=X_{1^{\prime} 1^{\prime}}^{k+1} w_{0}=0$. Тогда

$$
\left(X_{0^{\prime} 0^{\prime}}-X_{1^{\prime} 1^{\prime}}\right) \xi_{0}=\sum_{r=0}^{k-1} X_{0^{\prime} 0^{\prime}}^{r+1} X_{1^{\prime} 1^{\prime}}^{k-r} w_{0}-\sum_{r=1}^{k} X_{0^{\prime} 0^{\prime}}^{r} X_{1^{\prime} 1^{\prime}}^{k-r+1} w_{0}=0 .
$$

Вектор $X_{0^{\prime} 0^{\prime}}^{r} X_{1^{\prime} 1^{\prime}}^{k-r} w_{0}$ является вектором веса $(k-2 r, 2 r-k, 0, \ldots, 0)$, следовательно, $\left(H_{00}+H_{11}\right) X_{0^{\prime} 0^{\prime}}^{r} X_{1^{\prime} 1^{\prime}}^{k-r} w_{0}=0$ и $\left(H_{00}+H_{11}\right) \xi_{0}=0$. Применяя лемму 2.1 при $X_{\alpha}=X_{00}-X_{11}, X_{-\alpha}=X_{0^{\prime} 0^{\prime}}-X_{1^{\prime} 1^{\prime}}, H_{\alpha}=H_{00}+H_{11}$, получаем, что $\left(X_{00}-X_{11}\right) \xi_{0}=0$.

Г) Остается проверить, что $\xi_{0} \neq 0$. Пользуясь леммой 2.1 , получим

$$
\left\langle X_{0^{\prime} 0^{\prime}}^{r} X_{1^{\prime} 1^{\prime}}^{k-r} w_{0}, X_{0^{\prime} 0^{\prime}}^{r} X_{1^{\prime} 1^{\prime}}^{k-r} w_{0}\right\rangle=\left\langle X_{00}^{r} X_{0^{\prime} 0^{\prime}}^{r} X_{11}^{k-r} X_{1^{\prime} 1^{\prime}}^{k-r} w_{0}, w_{0}\right\rangle=(k !)^{2}\left\langle w_{0}, w_{0}\right\rangle
$$

Слагаемые в (2.20) ортогональны (так как имеют различные веса), следовательно,

$$
\left\langle\xi_{0}, \xi_{0}\right\rangle=(k+1)(k !)^{2}\left\langle w_{0}, w_{0}\right\rangle=k !(k+1) !\left\langle w_{0}, w_{0}\right\rangle .
$$

Пусть $v_{r}=\mathscr{D}^{r} v_{0}, A_{r}=\left\langle v_{r}, X_{1^{\prime} 2^{\prime}}^{r} v_{0}\right\rangle$. Индукцией по $r$ проверим, что числа $A_{r} \neq 0$ при $r \leqslant l$. Число $A_{0}=\left\langle v_{0}, v_{0}\right\rangle \neq 0$. Пользуясь $(2.17)$, получаем

$$
\left\langle v_{r}, X_{1^{\prime} 2^{\prime}}^{r} v_{0}\right\rangle=\left\langle\mathscr{D} v_{r-1}, X_{1^{\prime} 2^{\prime}}^{r} v_{0}\right\rangle=\left\langle v_{r-1}, \mathscr{D}^{*} X_{1^{\prime} 2^{\prime}}^{r} v_{0}\right\rangle
$$

где

$$
\mathscr{D}^{*}=H_{23^{\prime}} X_{12}+\sum_{d=3}^{n} X_{2 d^{\prime}} X_{1 d}-\sum_{d=2}^{n} X_{2 d} X_{1 d^{\prime}}
$$


Заметим, что (при $d \geqslant 3$ )

$$
X_{1 d} X_{1^{\prime} 2^{\prime}}^{r} v_{0}=\left(X_{1 d} X_{1^{\prime} 2^{\prime}}^{r}-X_{1^{\prime} 2^{\prime}}^{r} X_{1 d}\right) v_{0}=\left[X_{1 d}, X_{1^{\prime} 2^{\prime}}^{r}\right] v_{0}=r X_{2^{\prime} d} X_{1^{\prime} 2^{\prime}}^{r-1} v_{0}
$$

Далее,

$$
\begin{aligned}
X_{2 d^{\prime}} X_{1 d} X_{1^{\prime} 2^{\prime}}^{r} v_{0} & =r\left[X_{2 d^{\prime}}, X_{2^{\prime} d} X_{1^{\prime} 2^{\prime}}^{r-1}\right] v_{0} \\
& =r H_{2 d^{\prime}} X_{1^{\prime} 2^{\prime}}^{r-1} v_{0}-r(r-1) X_{2^{\prime} d} X_{1^{\prime} d^{\prime}} X_{1^{\prime} 2^{\prime}}^{r-2} v_{0} \\
& =r(l-r+1) X_{1^{\prime} 2^{\prime}}^{r-1} v_{0}-r(r-1) X_{2^{\prime} d} X_{1^{\prime} d^{\prime}} X_{1^{\prime} 2^{\prime}}^{r-2} v_{0}
\end{aligned}
$$

Из (2.23) следует, что

$$
\left\langle v_{r-1}, X_{2^{\prime} d} X_{1^{\prime} d^{\prime}} X_{1^{\prime} 2^{\prime}}^{r-2} v_{0}\right\rangle=\left\langle X_{2 d^{\prime}} v_{r-1}, X_{1^{\prime} d^{\prime}} X_{1^{\prime} 2^{\prime}}^{r-2} v_{0}\right\rangle=0
$$

Следовательно,

$$
\left\langle v_{r-1}, X_{2 d^{\prime}} X_{1 d} X_{1^{\prime} 2^{\prime}}^{r} v_{0}\right\rangle=r(l-r+1)\left\langle v_{r-1}, X_{1^{\prime} 2^{\prime}}^{r-1} v_{0}\right\rangle=r(l-r+1) A_{r-1} .
$$

Аналогично, при $d \geqslant 3$

$$
\left\langle v_{r-1}, X_{2 d} X_{1 d^{\prime}} X_{1^{\prime} 2^{\prime}}^{r} v_{0}\right\rangle=-r(l-r+1) A_{r-1}
$$

и

$$
\left\langle v_{r-1}, X_{22} X_{12^{\prime}} X_{1^{\prime} 2^{\prime}}^{r} v_{0}\right\rangle=-2 r(l-r+1) A_{r-1} .
$$

Кроме того,

$H_{23^{\prime}} X_{12} X_{1^{\prime} 2^{\prime}}^{r} v_{0}=r(k+2 l-r+1) H_{23^{\prime}} X_{1^{\prime} 2^{\prime}}^{r-1} v_{0}=r(l-r+1)(k+2 l-r+1) X_{1^{\prime} 2^{\prime}}^{r-1} v_{0}$.

Окончательно получаем, что

$$
A_{r}=\left\langle v_{r-1}, \mathscr{D}^{*} X_{1^{\prime} 2^{\prime}}^{r} v_{0}\right\rangle=r(l-r+1)(k+2 l-r+2 n-1) A_{r-1} \neq 0
$$

при $r \leqslant l$. Следовательно, $A_{l}=\left\langle w_{0}, X_{1^{\prime} 2^{\prime}}^{r} v_{0}\right\rangle \neq 0$ и $w_{0} \neq 0$. Тогда из $(2.27)$ следует, что и вектор $\xi_{0}$ не равен нулю.

ЗАмЕчаниЕ 2.5 . В ходе доказательства получено, что $X_{11} w_{0}=0$, где $w_{0}=$ $\mathscr{D}^{l} v_{0}, v_{0}$ - старший вектор пространства $E^{\lambda}, \lambda=(k, k, l, 0, \ldots, 0)$. 
СлЕДСТвИЕ 2.6. Пусть $0 \neq \xi_{0} \in E_{0}^{\lambda}, \quad F(x)$ - функиия на $M$, принимающая значения в $E^{\lambda}$ и удовлетворяющая условию (2.14). Тогда существует комплекснозначная функция $f(t), t \in \mathbb{R}$, для которой $F(\alpha(t) o)=f(t) \xi_{0}$.

Действительно, в предложении 2.3 показано, что вектор $F(\alpha(t) o)$ принадлежит $E_{0}^{\lambda}$, следовательно, он коллинеарен вектору $\xi_{0}$.

Пусть $\lambda=(k, k, l, 0, \ldots, 0) \in \Lambda_{0}, \delta_{1}=(1,1,0, \ldots, 0), \delta_{2}=(-1,-1,1,0, \ldots, 0)$. Если $\omega$ пробегает веса (2.11), то $\lambda+\omega$ может принадлежать $\Lambda_{0}$ только при $\omega=$ $\pm \omega_{01}= \pm \delta_{1}$ и $\omega= \pm \omega_{02^{\prime}}=\mp \delta_{2}$. Из предложения 2.2 следует, что в пространстве $P_{0}(\lambda, \mu)$ ненулевые операторы могут быть только при $\mu=\lambda \pm \delta_{1}$ или $\mu=\lambda \pm \delta_{2}$, если при этом соответствующие веса $\lambda \pm \delta_{1}$ или $\lambda \pm \delta_{2}$ являются доминантными (если вес $\mu$ не является доминантным, то будем считать, что $P_{0}(\lambda, \mu)=0$ ). Выберем какие-нибудь операторы (ненулевые, если соответствующее пространство $\left.P_{0}(\lambda, \mu) \neq 0\right)$ :

$$
\begin{array}{ll}
X_{+}^{(\lambda)} \in P_{0}\left(\lambda, \lambda+\delta_{1}\right), & X_{-}^{(\lambda)} \in P_{0}\left(\lambda, \lambda-\delta_{1}\right), \\
Y_{+}^{(\lambda)} \in P_{0}\left(\lambda, \lambda+\delta_{2}\right), & Y_{-}^{(\lambda)} \in P_{0}\left(\lambda, \lambda-\delta_{2}\right) .
\end{array}
$$

Из предложения 2.2 следует, что эти операторы определены с точностью до умножения на число. Тогда

$$
\begin{aligned}
X_{ \pm}^{(\lambda)}\left(\mathscr{F}_{\sigma}^{(\lambda)}\right) \subseteq \mathscr{F}_{\sigma}^{\left(\lambda \pm \delta_{1}\right)}, & Y_{ \pm}^{(\lambda)}\left(\mathscr{F}_{\sigma}^{(\lambda)}\right) \subseteq \mathscr{F}_{\sigma}^{\left(\lambda \pm \delta_{2}\right)} \\
X_{ \pm}^{(\lambda)}\left(\mathscr{F}_{\#}^{(\lambda)}\right) \subseteq \underset{\#}{\left(\lambda \pm \delta_{1}\right)}, & Y_{ \pm}^{(\lambda)}\left(\mathscr{F}_{\#}^{(\lambda)}\right) \subseteq \mathscr{F}_{\#}^{\left(\lambda \pm \delta_{2}\right)}
\end{aligned}
$$

ПРЕДЛОЖЕНИЕ 2.7. Пусть в каждом пространстве $\mathscr{F}_{\#}^{(\lambda)}, \lambda \in \Lambda_{0}$, выделено линейное подпространство $H_{\#}^{(\lambda)}$ так, что выполняются условия

$$
X_{ \pm}^{(\lambda)}\left(H_{\#}^{(\lambda)}\right) \subseteq H_{\#}^{\left(\lambda \pm \delta_{1}\right)}, \quad Y_{ \pm}^{(\lambda)}\left(H_{\#}^{(\lambda)}\right) \subseteq H_{\#}^{\left(\lambda \pm \delta_{2}\right)}
$$

Пусть $H$ - замыкание в $\mathscr{F}$ линейной оболочки всех функиий $\langle F(x), \xi\rangle$ для $F \in H_{\#}^{(\lambda)}, \quad \xi \in E^{\lambda}, \lambda \in \Lambda_{0}$.

Тогда Н будет инвариантным подпространством и соответствующие ему ячейки $H^{(\lambda)}$ совпадают с замыканием $H_{\#}^{(\lambda)}$ в $\mathscr{F}^{(\lambda)}$.

Доказательство сразу следует из предложения 2.4 в [1].

\section{§ 3. Вид оператора $\Delta$ в пространстве $\mathscr{F}(\lambda)$}

Пусть $F(x)$ - гладкая функция на $M$, принимающая значения в $E^{\lambda}, \lambda \in \Lambda_{0}$, и удовлетворяюшая условию (2.14). Тогда по следствию $2.5 F(\alpha(t) o)=f(t) \xi_{0}$, где $f(t)$ - комплекснозначная функция. Вектор $\xi_{0}$ можно выбрать так, чтобы $\left\|\xi_{0}\right\|=$ $\left\langle\xi_{0}, \xi_{0}\right\rangle^{1 / 2}=1$. Введем отображение

$$
D: F(x) \rightarrow f(t)
$$


Из (2.15) следует, что $F(x)$ однозначно восстанавливается по $f(t)$. Если $\Delta$ - оператор Лапласа-Бельтрами на $M$, то функция $(\Delta F)(x)$ также удовлетворяет условию (2.14). Поэтому действие оператора $\Delta$ можно выразить через функцию $f(t)$, т.е. найти оператор $D \Delta D^{-1}$ (для краткости будем его обозначать просто $\Delta$ ). Явный вид оператора $\Delta f$ будет найден в этом параграфе. Очевидно, что

$$
\Delta f(t)=\left\langle\Delta F(\alpha(t) o), \xi_{0}\right\rangle .
$$

Временно пусть $M$ - произвольное симметрическое пространство ранга 1 некомпактного типа. Группы $G$ и $K$ определяются так же, как в $\S 1, \mathfrak{g}_{0}$ и $\mathfrak{k}_{0}-$ соответствующие им алгебры Ли, $\mathfrak{g}_{0}=\mathfrak{k}_{0}+\mathfrak{p}_{0}$ - разложение Картана, $\mathfrak{a}_{0}$ - максимальное абелево подпространство в $\mathfrak{p}_{0}\left(\operatorname{dim} \mathfrak{a}_{0}=1\right)$. Через $\mathfrak{g}, \mathfrak{k}, \mathfrak{p}$ и $\mathfrak{a}$ обозначим комплексификации пространств $\mathfrak{g}_{0}, \mathfrak{k}_{0}, \mathfrak{p}_{0}$ и $\mathfrak{a}_{0}$ соответственно. Пусть $\mathfrak{k}_{1}-$ централизатор подалгебры $\mathfrak{a}$ в $\mathfrak{k}, \mathfrak{l}$ - ортогональное дополнение к $\mathfrak{k}_{1}$ в $\mathfrak{k}$ относительно формы Киллинга $B(B-$ форма Киллинга на алгебре $\mathfrak{g})$.

Множество $\Pi^{+}$ограниченных на $\mathfrak{a}$ положительных корней для симметрического пространства ранга 1 состоит из элементов $\beta$ и $2 \beta$ с некоторыми кратностями $m_{\beta}$ и $m_{2 \beta}$. Для $\gamma \in \Pi^{+}$пусть

$$
\mathfrak{k}_{\gamma}=\left\{T \in \mathfrak{k}:(\operatorname{ad} h)^{2} T=\gamma^{2}(H) T \quad \forall H \in \mathfrak{g}\right\} .
$$

Известно $[17$, с. 346$]$, что $\operatorname{dim} \mathfrak{k}_{\gamma}=m_{\gamma}$, где $m_{\gamma}-$ кратность корня $\gamma$, и что $\mathfrak{l}=\mathfrak{k}_{\beta}+$ $\mathfrak{k}_{2 \beta}$. Пусть $T_{1}^{\gamma}, \ldots, T_{m_{\gamma}}^{\gamma}$ - базис в $\mathfrak{k}_{\gamma}$, ортонормированньй относительно формы $(-B)$. Положим

$$
\omega_{\gamma}=\sum_{j=1}^{m_{\gamma}}\left(T_{j}^{\gamma}\right)^{2} \in U(\mathfrak{k}) .
$$

Выберем базисный вектор $I_{0} \in \mathfrak{a}$ так, чтобы $\beta\left(I_{0}\right)=1$. Тогда

$$
\mathfrak{k}_{\beta}=\left\{T \in \mathfrak{k}:\left(\operatorname{ad} I_{0}\right)^{2} T=T\right\}, \quad \mathfrak{k}_{2 \beta}=\left\{T \in \mathfrak{k}:\left(\operatorname{ad} I_{0}\right)^{2} T=4 T\right\} .
$$

Положим $\alpha(t)=\exp \left(t I_{0}\right) \in G$. Пусть $A_{\gamma}-$ корневой вектор, т.е. $A_{\gamma} \in \mathfrak{a}$ и $B\left(A_{\gamma}, H\right)=\gamma(H)$ при $H \in \mathfrak{a}$. В нашем случае $A_{\beta}=B\left(I_{0}, I_{0}\right)^{-1} I_{0}, A_{2 \beta}=2 A_{\beta}$.

Пусть $T^{\lambda}$ - произвольное конечномерное представление группы $K$ в пространстве $E^{\lambda}$. Соответствуюшие представления алгебры Ли k и универсальной обертываюшей алгебры $U(\mathfrak{k})$ также будем обозначать $T^{\lambda}$. Пусть $F(x)$ - гладкая функция на $M$, принимающая значения в $E^{\lambda}$ и удовлетворяюшая условию (2.14). Из вида оператора Бельтрами-Лапласа в полярных координатах на симметрическом пространстве $M[17$, гл. 2 , теорема 5.24$]$ следует, что

$$
\begin{aligned}
\Delta F(\alpha(t) o)= & \frac{1}{B\left(I_{0}, I_{0}\right)}\left[\partial_{t}^{2} F(\alpha(t) o)+m_{\beta} \operatorname{cth} t \partial_{t} F(\alpha(t) o)\right. \\
& \left.+2 m_{2 \beta} \operatorname{cth} 2 t \partial_{t} F(\alpha(t) o)\right] \\
& +\left[(\operatorname{sh} t)^{-2} T^{\lambda}\left(\omega_{\beta}\right)+(\operatorname{sh} 2 t)^{-2} T^{\lambda}\left(\omega_{2 \beta}\right)\right] F(\alpha(t) o),
\end{aligned}
$$


где $\partial_{t}-$ дифференщирование по $t$.

Вернемся к случаю, когда $M=\operatorname{Sp}(1, n) / \operatorname{Sp}(1) \times \operatorname{Sp}(n)$. Тогда $m_{\beta}=4(n-1)$, $m_{2 \beta}=3$. Отметим, что на алгебре Ли $\operatorname{sp}(n)$ форма Киллинга имеет вид $B(X, Y)=$ $2(n+1) \operatorname{tr}(X Y)\left[12\right.$, c.88]. Соответственно на алгебре Ли $\mathfrak{g}_{0}=\operatorname{sp}(1, n)$ и на ее комплексной оболочке $\mathfrak{g}=\operatorname{sp}(n+1)$ форма Киллинга имеет вид $B(X, Y)=$ $2(n+2) \operatorname{tr}(X Y)$. Как и в $\S 2$, в нашем случае $I_{0}=X_{01^{\prime}}+X_{10^{\prime}}$. Тогда $B\left(I_{0}, I_{0}\right)=$ $8(n+2)$. Положим $\tilde{\Delta}=B\left(I_{0}, I_{0}\right) \Delta=8(n+2) \Delta$.

Введем новый базис в алгебре $\mathfrak{g}$. Пусть $H_{j}=i H_{j j} ; \quad Y_{j r}=X_{j r}-X_{j^{\prime} r^{\prime}}$, $Z_{j r}=i\left(X_{j r}+X_{j^{\prime} r^{\prime}}\right)$ при $0 \leqslant j \leqslant r \leqslant n ; \quad Y_{j r^{\prime}}=X_{j r^{\prime}}-X_{j^{\prime} r}, \quad Z_{j r^{\prime}}=$ $i\left(X_{j r^{\prime}}+X_{j^{\prime} r}\right)$ при $0 \leqslant j<r \leqslant n$. Эти матрищы образуют ортогональный базис в $\mathfrak{g}$ (относительно формы Киллинга) и, кроме того, $B\left(H_{j}, H_{j}\right)=-4(n+2)$, $B\left(Y_{j r}, Y_{j r}\right)=B\left(Z_{j r}, Z_{j r}\right)=B\left(Y_{j r^{\prime}}, Y_{j r^{\prime}}\right)=B\left(Z_{j r^{\prime}}, Z_{j r^{\prime}}\right)=-8(n+2)$ при $j \neq r$ и $B\left(Y_{j j}, Y_{j j}\right)=B\left(Z_{j j}, Z_{j j}\right)=-4(n+2)$.

Легко видеть, что базис пространства $\mathfrak{k}_{\beta}$ образуют матрицы

$$
Y_{1 j}, \quad Z_{1 j}, \quad Y_{1 j^{\prime}}, \quad Z_{1 j^{\prime}} \text { при } 2 \leqslant j \leqslant n .
$$

Тогда

$$
\omega_{\beta}=(8(n+2))^{-1}\left(\sum_{2 \leqslant j \leqslant n} Y_{1 j}^{2}+Z_{1 j}^{2}+Y_{1 j^{\prime}}^{2}+Z_{1 j^{\prime}}^{2}\right) .
$$

Ортогональный базис пространства $\mathfrak{k}_{2 \beta}$ образуют матрицы

$$
H_{1}-H_{0}, \quad Y_{00}+Y_{11}, \quad Z_{00}+Z_{11}
$$

При этом

$$
\omega_{2 \beta}=(8(n+2))^{-1}\left(\left(H_{0}-H_{1}\right)^{2}+\left(Y_{00}+Y_{11}\right)^{2}+\left(Z_{00}+Z_{11}\right)^{2}\right) .
$$

Напомним некоторые факты из теории полупростых алгебр Ли (см. [13]). Пусть $\mathfrak{g}$ - полупростая алгебра Ли, $B$ - форма Киллинга; $X_{1}, \ldots, X_{N}$ - базис в $\mathfrak{g}$, $X^{1}, \ldots, X^{N}$ - двойственный базис в $\mathfrak{g}$, т.е. $B\left(X_{j}, X^{r}\right)=\delta_{j}^{r}$, где $\delta_{j}^{r}$ - символ Кронекера. Элемент $c=X_{1} X^{1}+\cdots+X_{N} X^{N} \in U(\mathfrak{g})$ называется әлементом Казимира. Пусть $\mathfrak{h}$ - подалгебра Картана в $\mathfrak{g}, R$ - система корней алгебры $\mathfrak{g}$ относительно $\mathfrak{h}, R_{+}-$множество положительных корней. Через $\langle$,$\rangle обозначим форму$ на $\mathfrak{h}^{*}$, являющуюся обратной формой для ограничения формы $B$ на $\mathfrak{h}$. Пусть $V-$ неприводимьй конечномерный $\mathfrak{g}$-модуль со старшим весом $\lambda$. Тогда для любого $\xi \in V$

$$
c \xi=\langle\lambda, \lambda+2 \rho\rangle \xi
$$

где

$$
\rho=\frac{1}{2} \sum_{\alpha \in R_{+}} \alpha
$$

Вернемся к нашему случаю: $E^{\lambda}$ - неприводимый k-модуль со старшим весом $\lambda=(k, k, l, 0, \ldots, 0) \in \Lambda_{0}$. Вектор $\xi_{0} \in E^{\lambda}$ определяется условиями $\mathfrak{k}_{1} \xi_{0}=0$ и 
$\left\|\xi_{0}\right\|=1$. Как и в $\S 2$, будем отождествлять $\operatorname{sp}(1)$ с подалгеброй в $\mathfrak{k}$, состоящей из матрищ (2.5) при $A_{1}=B_{1}=C_{1}=0, \mathrm{a} \operatorname{sp}(n)$ с подалгеброй в $\mathfrak{k}$, состоящей из матрищ (2.5) при $a=b=c=0$. Пусть $c_{1}$ - элемент Казимира алгебры $\operatorname{sp}(1)$, a $c_{2}$ - элемент Казимира алгебры $\operatorname{sp}(n)$. Заметим, что k-модуль $E^{\lambda}$ изоморфен тензорному произведению $E^{\lambda_{1}} \otimes E^{\lambda_{2}}$, где $E^{\lambda_{1}}$ - неприводимый $\operatorname{sp}(1)$-модуль со старшим весом $\lambda_{1}=(k)$, а $E^{\lambda_{2}}$ - неприводимый $\operatorname{sp}(n)$-модуль со старшим весом $\lambda_{2}=(k, l, 0, \ldots, 0)$. Следовательно, для любого $\xi \in E^{\lambda}$ имеем $c_{1} \xi=\left\langle\lambda_{1}, \lambda_{1}+2 \rho_{2}\right\rangle \xi$ и $c_{2} \xi=\left\langle\lambda_{2}, \lambda_{2}+2 \rho_{2}\right\rangle \xi$, где $\rho_{1}$ и $\rho_{2}-$ полусуммы положительных корней алгебр Ли $\operatorname{sp}(1)$ и $\mathrm{sp}(n)$ соответственно. Нетрудно видеть, что в явном виде

$$
\begin{gathered}
\left\langle\lambda_{1}, \lambda_{1}+2 \rho_{1}\right\rangle=8^{-1} k(k+2), \\
\left\langle\lambda_{2}, \lambda_{2}+2 \rho_{2}\right\rangle=(4(n+1))^{-1}[(k+l)(k+l+2 n)+l(l+2 n-2)] .
\end{gathered}
$$

В алгебре Ли $\operatorname{sp}(1)$ базис образуют матрицы $H_{0}, Y_{00}, Z_{00}$, элемент Казимира равен $c_{1}=-8^{-1}\left(H_{0}^{2}+Y_{00}^{2}+Z_{00}^{2}\right)$. Так как векторы $H_{0}+H_{1}, Y_{00}-Y_{11}, Z_{00}-Z_{11}$ принадлежат $\mathfrak{k}_{1}$, то

$$
\left[\left(H_{0}-H_{1}\right)^{2}+\left(Y_{00}+Y_{11}\right)^{2}+\left(Z_{00}+Z_{11}\right)^{2}\right] \xi_{0}=4\left(H_{0}^{2}+Y_{00}^{2}+Z_{00}^{2}\right) \xi_{0}
$$

и, следовательно,

$$
\omega_{2 \beta} \xi_{0}=-4(n+2)^{-1} c_{1} \xi_{0}=-(2(n+2))^{-1} k(k+2) .
$$

В алгебре Ли $\operatorname{sp}(n)$ базис образуют матрицы $H_{j}(1 \leqslant j \leqslant n), Y_{j r}, Z_{j r}(1 \leqslant j \leqslant$ $r \leqslant n)$ и $Y_{j r^{\prime}}, Z_{j r^{\prime}}(1 \leqslant j<r \leqslant n)$. Из этих матриц не принадлежат подалгебре $\mathfrak{k}_{1}$ только матрицы $H_{1}, Y_{1 r}, Z_{1 r}, Y_{1 r^{\prime}}, Z_{1 r^{\prime}}$. Элемент Казимира на алгебре Ли $\operatorname{sp}(n)$ имеет вид

$$
c_{2}=-\frac{1}{8(n+1)}\left[2 \sum_{1 \leqslant j \leqslant n}\left(H_{j}^{2}+Y_{j j}^{2}+Z_{j j}^{2}\right)+\sum_{1 \leqslant j<r \leqslant n}\left(Y_{j r}^{2}+Z_{j r}^{2}+Y_{j r^{\prime}}^{2}+Z_{j r^{\prime}}^{2}\right)\right] .
$$

Тогда

$$
c_{2} \xi_{0}=-\frac{1}{8(n+1)}\left[2\left(H_{1}^{2}+Y_{11}^{2}+Z_{11}^{2}\right)+\sum_{2 \leqslant r \leqslant n}\left(Y_{1 r}^{2}+Z_{1 r}^{2}+Y_{1 r^{\prime}}^{2}+Z_{1 r^{\prime}}^{2}\right)\right] \xi_{0} .
$$

Заметим еше, что из $H_{0}+H_{1}, Y_{00}-Y_{11}, Z_{00}-Z_{11} \in \mathfrak{k}_{1}$ следует

$$
\left(H_{1}^{2}+Y_{11}^{2}+Z_{11}^{2}\right) \xi_{0}=\left(H_{0}^{2}+Y_{00}^{2}+Z_{00}^{2}\right) \xi_{0} .
$$

Из $(3.9),(3.10)$ и (3.8) получим, что

$$
c_{2} \xi_{0}=-\frac{(n+2)}{(n+1)}\left[\frac{1}{2} \omega_{2 \beta}+\omega_{\beta}\right] \xi_{0},
$$


следовательно,

$$
\begin{aligned}
\omega_{\beta} \xi_{0} & =-\frac{(n+1)}{(n+2)} c_{2} \xi_{0}-\frac{1}{2} \omega_{2 \beta} \xi_{0} \\
& =-(4(n+2))^{-1}[(k+l)(k+l+2 n)+l(l+2 n-2)-k(k+2)] \xi_{0} .
\end{aligned}
$$

Из (3.2), (3.8) и (3.11) с учетом того, что

$$
F(\alpha(t) o)=f(t) \xi_{0}, \quad \tilde{\Delta}=B\left(I_{0}, I_{0}\right) \Delta=8(n+2) \Delta,
$$

получаем окончательный ответ

$$
\begin{aligned}
\tilde{\Delta} f(t)= & \frac{d^{2} f}{d t^{2}}(t)+4(n-1) \operatorname{cth} t \frac{d f}{d t}(t)+6 \operatorname{cth} 2 t \frac{d f}{d t}(t)-4 k(k+2)(\operatorname{sh} 2 t)^{-2} f(t) \\
& -2[(k+l)(k+l+2 n)+l(l+2 n-2)-k(k+2)](\operatorname{sh} t)^{-2} f(t) .
\end{aligned}
$$

\section{§4. Вычисление операторов $X_{ \pm}^{(\lambda)}, Y_{ \pm}^{(\lambda)}$ и доказательство теорем I, II}

Пусть $F(x) \in \mathscr{F}_{\sigma}^{(\lambda)}, \lambda \in \Lambda_{0}$. Как и в $\S 3$, определим отображение

$$
D=D^{\lambda}: F(x) \rightarrow f(t),
$$

где

$$
F(\alpha(t) o)=f(t) \xi_{0}
$$

Если оператор $X$ принадлежит $P_{0}(\lambda, \mu), \mu \in \Lambda_{0}$, то $X$ переводит пространство $\mathscr{F}_{\sigma}^{(\lambda)}$ в $\mathscr{F}_{\sigma}^{(\mu)}$, поэтому его действие можно выразить через функцию $f(t)$, т.е. найти $D^{\mu} X\left(D^{\lambda}\right)^{-1}$. Для краткости этот оператор будем обозначать просто через $X . \mathrm{B}$ этом параграфе будет найден явный вид таких операторов.

В качестве базиса пространства $\mathfrak{p}$ возьмем матрицы $p_{1}, p_{2}, \ldots, p_{4 n}$, определенные следуюшим образом:

$$
\begin{aligned}
p_{4 j-3} & =X_{0 j^{\prime}}+X_{0^{\prime} j}, \quad p_{4 j-2}=i\left(X_{0 j^{\prime}}-X_{0^{\prime} j}\right), \\
p_{4 j-1} & =X_{0 j}+X_{0^{\prime} j^{\prime}}, \quad p_{4 j}=i\left(X_{0 j}-X_{0^{\prime} j^{\prime}}\right), \quad j=1,2, \ldots, n .
\end{aligned}
$$

Матрицы $p_{j}$ образуют ортогональный базис (относительно формы Киллинга) в $\mathfrak{p}$ и $B\left(p_{j}, p_{j}\right)=8(n+2)$. Отметим также, что все $p_{j} \in \mathfrak{p}_{0}$ и $p_{1}=I_{0}$.

Рассуждая как в $\S 5$ работы [1], получаем, что для любых матриц $p_{j}$ и $p_{r}$ найдется элемент $u \in K$ такой, что $p_{r}=u p_{j} u^{-1}$, а также, что матрища оператоpa ad $\left.k\right|_{\mathfrak{k}}$ в базисе $p_{1}, \ldots, p_{4 n}$ вещественна и кососимметрична для любого $k \in \mathfrak{k}_{0}$. При $k \in \mathfrak{k}$ матрица оператора $\left.\operatorname{ad} k\right|_{\mathfrak{k}}$ в базисе $p_{1}, \ldots, p_{4 n}$ также будет кососиммет-

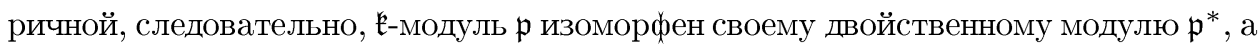


базис $p_{1}, \ldots, p_{4 n}$ совпадает со своим двойственным базисом. Отметим также, что матрищы операторов $\left.\mathrm{Ad}(u)\right|_{\mathfrak{k}}$ в базисе $p_{1}, \ldots, p_{4 n}$ вешественны и ортогональны при $u \in K$.

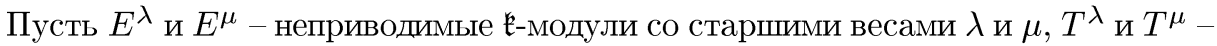
возникающие в $E^{\lambda}$ и $E^{\mu}$ представления групшы $K$. Пусть $\varphi: \mathfrak{p} \rightarrow \operatorname{Hom}\left(E^{\mu}, E^{\lambda}\right)-$ гомоморфизм k-модулей, $\alpha_{j}=\varphi\left(p_{j}\right), \alpha_{j}^{*}-$ сопряженный оператор. Тогда $\alpha_{j}^{*} \in$ $\operatorname{Hom}\left(E^{\lambda}, E^{\mu}\right)$. Оператор $X=L(\varphi)$ задается формулой $(2.3)$ и действует из пространства $\mathscr{F}_{\sigma}^{(\lambda)}$ в $\mathscr{F}_{\sigma}^{(\mu)}$.

Гомоморфизм k-модулей $\varphi$ индуцирует сплетающий оператор для соответствуюших представлений групшы $K$. Тогда если

$$
\operatorname{Ad}(u) p_{j}=u p_{j} u^{-1}=\sum c_{j r}(u) p_{r}
$$

TO

$$
T^{\lambda}(u) \alpha_{j} T^{\mu}\left(u^{-1}\right)=\sum c_{j r}(u) \alpha_{r},
$$

а так как операторы $T^{\lambda}(u)$ и $T^{\mu}(u)$ унитарны и числа $c_{j r}(u)$ принадлежат $\mathbb{R}$, то и

$$
T^{\mu}(u) \alpha_{j}^{*} T^{\lambda}\left(u^{-1}\right)=\sum c_{j r}(u) \alpha_{r}^{*}
$$

Повторяя рассуждения леммы 5.2 в [1], получаем, что при $F \in \mathscr{F}_{\sigma}^{(\lambda)}$

$$
(X F)(x)=\left.4 n \frac{d}{d s}\left(\int T^{\mu}(u) \alpha_{1}^{*} T^{\lambda}\left(u^{-1}\right) F\left(u \alpha(s) u^{-1} x\right) d u\right)\right|_{s=0} .
$$

Здесь и в дальнейших формулах $d u$ - элемент меры Хаара на групше $K$ и интегралы по $d u$ берутся по группе $K$.

Напомним, что $\mathfrak{l}=\mathfrak{k}_{1}^{\perp}-$ ортогональное дополнение к $\mathfrak{k}_{1}$ в $\mathfrak{k}$ относительно формы Киллинга. Пусть $\mathfrak{l}_{0}=\mathfrak{l} \cap \mathfrak{k}_{0}$. Отображение $\tau: \mathfrak{k}_{0} \times \mathbb{R} \times \mathfrak{l}_{0} \rightarrow G$ определим формулой

$$
\tau(k, t, q)=(\exp k) \alpha(t)(\exp q)
$$

где $q \in \mathfrak{l}_{0}, t \in \mathbb{R}, k \in \mathfrak{k}_{0}$. Пусть $t \neq 0$. Нетрудно найти дифференциал отображения $\tau$ в точке $(0, t, 0)$ и показать, что он невырожден. Следовательно, отображение $\tau$ является диффеоморфизмом некоторой окрестности точки $(0, t, 0)$ в $\mathfrak{k}_{0} \times \mathbb{R} \times \mathfrak{l}_{0}$ на окрестность точки $\alpha(t)$ в $G$. Тогда при $s$, достаточно близких к нулю,

$$
u \alpha(s) u^{-1} \alpha(t)=v \alpha(\tilde{t}) w
$$

где $v=\exp p, w=\exp q, \tau^{-1}\left(u \alpha(s) u^{-1} \alpha(t)\right)=(k, \tilde{t}, q)$. При этом $v, \tilde{t}, w$ гладко зависят от $s$ и $\tilde{t}(0)=t$. Пусть

$$
t_{1}=\left.\frac{d \tilde{t}}{d s}\right|_{s=0}, \quad v_{1}=\left.\frac{d v}{d s}\right|_{s=0}, \quad w_{1}=\left.\frac{d w}{d s}\right|_{s=0}
$$


Тогда $v_{1} \in \mathfrak{k}_{0}, w_{1} \in \mathfrak{l}_{0}$. Продифференцируем равенство (4.4) по $s$ и положим $s=0$. Получим

$$
u I_{0} u^{-1} \alpha(t)=v_{1} \alpha(t)+t_{1} I_{0} \alpha(t)+\alpha(t) w_{1},
$$

или, эквивалентно,

$$
u I_{0} u^{-1}=v_{1}+t_{1} I_{0}+\alpha(t) w_{1} \alpha(-t)
$$

В пространстве $\mathfrak{l}$ базис образуют матрицы (3.3) и (3.4). Будем обозначать эти базисные векторы $q_{2}, q_{3}, \ldots, q_{4 n}$, причем пусть

$$
q_{2}=H_{1}-H_{0}, \quad q_{3}=Y_{00}+Y_{11}, \quad q_{4}=Z_{00}+Z_{11},
$$

а при $r \geqslant 2$

$$
q_{4 r-3}=Y_{1 r^{\prime}}, \quad q_{4 r-2}=Z_{1 r^{\prime}}, \quad q_{4 r-1}=Y_{1 r}, \quad q_{4 r}=Z_{1 r} .
$$

Легко видеть, $\quad$ что $\quad \alpha(t) q_{j} \alpha(-t)=(\operatorname{ch} 2 t) q_{j}+(\operatorname{sh} 2 t) p_{j} \quad$ при $\quad j=2,3,4 \quad$ и $\alpha(t) q_{j} \alpha(-t)=(\operatorname{ch} t) q_{j}+(\operatorname{sh} t) p_{j}$ при $j \geqslant 5$.

Так как $I_{0}=p_{1}$, то

$$
u I_{0} u^{-1}=\operatorname{Ad}(u) p_{1}=\sum_{1 \leqslant j \leqslant 4 n} c_{1 j}(u) p_{j}
$$

Матрицу $w_{1} \in \mathfrak{l}_{0}$ можно разложить по базису $q_{j}$ :

$$
w_{1}=\sum_{2 \leqslant j \leqslant 4 n} x_{j} q_{j}
$$

где $x_{j} \in \mathbb{R}$. Тогда из (4.6) получаем, что

$$
\begin{aligned}
& \sum_{1 \leqslant j \leqslant 4 n} c_{1 j}(u) p_{j}=\left(v_{1}+\left(x_{2} \operatorname{ch} 2 t\right) q_{2}+\left(x_{3} \operatorname{ch} 2 t\right) q_{3}+\left(x_{4} \operatorname{ch} 2 t\right) q_{4}+\sum_{5 \leqslant j \leqslant 4 n}\left(x_{j} \operatorname{ch} t\right) q_{j}\right) \\
& \quad+\left(t_{1} p_{1}+\left(x_{2} \operatorname{sh} 2 t\right) p_{2}+\left(x_{3} \operatorname{sh} 2 t\right) p_{3}+\left(x_{4} \operatorname{sh} 2 t\right) p_{4}+\sum_{5 \leqslant j \leqslant 4 n}\left(x_{j} \operatorname{sh} t\right) p_{j}\right) .
\end{aligned}
$$

Из (4.8) получаем (приравнивая коэффициенты при $p_{j}$ ), что $t_{1}=c_{11}, x_{j}=$ $(\operatorname{sh} t)^{-1} c_{1 j}$ при $j=2,3,4$ и $x_{j}=(\operatorname{sh} t)^{-1} c_{1 j}$ при $j \geqslant 5$. Тогда

$$
\begin{aligned}
& v_{1}=-(\operatorname{cth} 2 t)\left(c_{12} q_{2}+c_{13} q_{3}+c_{14} q_{4}\right)-(\operatorname{cth} t) \sum_{5 \leqslant j \leqslant 4 n} c_{1 j} q_{j}, \\
& w_{1}=(\operatorname{sh} 2 t)^{-1}\left(c_{12} q_{2}+c_{13} q_{3}+c_{14} q_{4}\right)+(\operatorname{sh} t)^{-1} \sum_{5 \leqslant j \leqslant 4 n} c_{1 j} q_{j} .
\end{aligned}
$$


Напомним, что для функции $F \in \mathscr{F}_{\sigma}^{(\lambda)}$ комплекснозначные функции $f(t)$ и $(X f)(t)$ определяются из соотношений

$$
F(\alpha(t) o)=f(t) \xi_{0}, \quad(X F)(\alpha(t) o)=(X f)(t) \tilde{\xi}_{0},
$$

где вектор $\xi_{0} \in E^{\lambda}$ удовлетворяет условиям $\mathfrak{k}_{1} \xi_{0}=0$ и $\left\|\xi_{0}\right\|=1$, а $\tilde{\xi}_{0}$ - аналогичньй вектор пространства $E^{\mu}$, т.е. $\mathfrak{k}_{1} \tilde{\xi}_{0}=0$ и $\left\|\tilde{\xi}_{0}\right\|=1$. Отметим, что

$$
f(t)=\left\langle F(\alpha(t) o), \xi_{0}\right\rangle, \quad(X f)(t)=\left\langle X F(\alpha(t) o), \tilde{\xi}_{0}\right\rangle .
$$

Лемма 4.1. Имеем

$$
(X f)(t)=A \frac{d f}{d t} f(t)-3 B(\operatorname{cth} 2 t) f(t)-4(n-1) C(\operatorname{cth} t) f(t),
$$

२дe

$$
A=\left\langle\alpha_{1}^{*} \xi_{0}, \tilde{\xi}_{0}\right\rangle, \quad B=\left\langle\alpha_{2}^{*}\left(q_{2} \xi_{0}\right), \tilde{\xi}_{0}\right\rangle, \quad C=\left\langle\alpha_{5}^{*}\left(q_{5} \xi_{0}\right), \tilde{\xi}_{0}\right\rangle
$$

ДоКАЗАТЕЛЬСТво. Из (4.4) следует, что

$$
F\left(u \alpha(s) u^{-1} \alpha(t) o\right)=T^{\lambda}(v) F(\alpha(\tilde{t}) o)=T^{\lambda}(v)\left(f(\tilde{t}) \xi_{0}\right) .
$$

Тогда

$$
\left.\frac{d F}{d s}\left(u \alpha(s) u^{-1} \alpha(t) o\right)\right|_{s=0}=t_{1} \frac{d f}{d t}(t) \cdot \xi_{0}+f(t)\left(v_{1} \xi_{0}\right) .
$$

Из (4.2) следует, что

$$
T^{\mu}(u) \alpha_{1}^{*} T^{\lambda}\left(u^{-1}\right)=\sum c_{1 j}(u) \alpha_{j}^{*} .
$$

Так как функции $c_{1 j}(u)$ являются матричными элементами неприводимого унитарного представления группы $K$ в пространстве p и $c_{1 j} \in \mathbb{R}$, то из соотношений ортогональности следует, что

$$
\int c_{1 j}(u) c_{1 r}(u) d u=(4 n)^{-1} \delta_{j r}
$$

где $\delta_{j r}$ - символ Кронекера. Умножим (4.14) на (4.13) и проинтегрируем по $u$, пользуясь соотношениями ортогональности. Тогда с учетом (4.3)

$$
\begin{aligned}
(X F)(\alpha(t) o)= & \frac{d f}{d t}(t) \cdot \alpha_{1}^{*} \xi_{0}-f(t)(\operatorname{cth} 2 t)\left(\alpha_{2}^{*} q_{2} \xi_{0}+\alpha_{3}^{*} q_{3} \xi_{0}+\alpha_{4}^{*} q_{4} \xi_{0}\right) \\
& -f(t)(\operatorname{cth} t) \sum_{5 \leqslant j \leqslant 4 n} \alpha_{j}^{*} q_{j} \xi_{0} .
\end{aligned}
$$

Докажем, что

$$
\left\langle\alpha_{2}^{*} q_{2} \xi_{0}, \tilde{\xi}_{0}\right\rangle=\left\langle\alpha_{3}^{*} q_{3} \xi_{0}, \tilde{\xi}_{0}\right\rangle=\left\langle\alpha_{4}^{*} q_{4} \xi_{0}, \tilde{\xi}_{0}\right\rangle
$$


и

$$
\left\langle\alpha_{j}^{*} q_{j} \xi_{0}, \tilde{\xi}_{0}\right\rangle=\left\langle\alpha_{5}^{*} q_{5} \xi_{0}, \tilde{\xi}_{0}\right\rangle \quad \text { при } \quad j \geqslant 5 .
$$

Определим элементы $k_{3}, k_{4}$ и $k_{6}, k_{7}, \ldots, k_{4 n}$ из алгебры Ли $\mathfrak{k}_{1}$ следующим обра30M:

$$
\begin{gathered}
k_{3}=\frac{1}{2}\left(Z_{11}-Z_{00}\right), \quad k_{4}=\frac{1}{2}\left(Y_{00}-Y_{11}\right), \\
k_{6}=H_{0}+H_{1}, \quad k_{7}=-Y_{22}, \quad k_{8}=-Z_{22},
\end{gathered}
$$

а при $j \geqslant 3$

$$
k_{4 j-3}=-Y_{2 j^{\prime}}, \quad k_{4 j-2}=-Z_{2 j^{\prime}}, \quad k_{4 j-1}=-Y_{2 j}, \quad k_{4 j}=-Z_{2 j} .
$$

Легко видеть, что

$$
\begin{array}{llll}
{\left[k_{r}, p_{2}\right]=p_{r},} & {\left[k_{r}, q_{r}\right]=-q_{2}} & \text { при } & r=3,4, \\
{\left[k_{r}, p_{5}\right]=p_{r},} & {\left[k_{r}, q_{r}\right]=-q_{5}} & \text { при } & r=6,7, \ldots, 4 n .
\end{array}
$$

По определению гомоморфизма $\varphi$

$$
\varphi([k, p]) \xi=k(\varphi(p) \xi)-\varphi(p)(k \xi)
$$

для любых $k \in \mathfrak{k}, p \in \mathfrak{p}, \xi \in E^{\mu}$. Для краткости формулу (4.16) будем также записывать как

$$
\varphi([k, p])=[k, \varphi(p)] .
$$

Отметим также, что $k_{j} \xi_{0}=0, k_{j} \tilde{\xi}_{0}=0$ и $k_{j}^{*}=-k_{j}$. Пусть $j=3$ или $j=4$; тогда

$$
\begin{aligned}
\left\langle\alpha_{j}^{*} q_{j} \xi_{0}, \tilde{\xi}_{0}\right\rangle & =\left\langle q_{j} \xi_{0}, \alpha_{j} \tilde{\xi}_{0}\right\rangle=\left\langle q_{j} \xi_{0}, \varphi\left(p_{j}\right) \tilde{\xi}_{0}\right\rangle \\
& =\left\langle q_{j} \xi_{0},\left(k_{j} \varphi\left(p_{2}\right)-\varphi\left(p_{2}\right) k_{j}\right) \tilde{\xi}_{0}\right\rangle=\left\langle q_{j} \xi_{0}, k_{j} \alpha_{2} \tilde{\xi}_{0}\right\rangle \\
& =-\left\langle k_{j} q_{j} \xi_{0}, \alpha_{2} \tilde{\xi}_{0}\right\rangle=-\left\langle\left[k_{j}, q_{j}\right] \xi_{0}, \alpha_{2} \tilde{\xi}_{0}\right\rangle=\left\langle q_{2} \xi_{0}, \alpha_{2} \tilde{\xi}_{0}\right\rangle=\left\langle\alpha_{2}^{*} q_{2} \xi_{0}, \tilde{\xi}_{0}\right\rangle .
\end{aligned}
$$

Аналогично проверяется, что $\left\langle\alpha_{j}^{*} q_{j} \xi_{0}, \tilde{\xi}_{0}\right\rangle=\left\langle\alpha_{5}^{*} q_{5} \xi_{0}, \tilde{\xi}_{0}\right\rangle$ при $j \geqslant 5$. Умножив (4.15) скалярно на $\tilde{\xi}_{0}$, получим (4.12).

По лемме 4.1 для нахождения явного вида оператора $X \in P_{0}(\lambda, \mu)$ необходимо найти три коэффициента: $A, B, C$. Следующая лемма упрощает нахождение этих коэффициентов. Напомним, что $p_{1}=X_{01^{\prime}}+X_{0^{\prime} 1}, p_{2}=i\left(X_{01^{\prime}}-X_{0^{\prime} 1}\right), p_{5}=X_{02^{\prime}}+$ $X_{0^{\prime} 2}, q_{2}=i\left(H_{11}-H_{00}\right), q_{5}=X_{12^{\prime}}-X_{1^{\prime} 2}$ и $\alpha_{j}=\varphi\left(p_{j}\right)$.

ЛЕМма 4.2. Имеем

$$
\begin{aligned}
& A=2\left\langle\xi_{0}, \varphi\left(X_{01^{\prime}}\right) \tilde{\xi}_{0}\right\rangle, \\
& B=4\left\langle H_{11} \xi_{0}, \varphi\left(X_{01^{\prime}}\right) \tilde{\xi}_{0}\right\rangle, \\
& C=2\left\langle X_{12^{\prime}} \xi_{0}, \varphi\left(X_{02^{\prime}}\right) \tilde{\xi}_{0}\right\rangle .
\end{aligned}
$$


ДоКАЗАТЕЛЬСТВО. Основным элементом следуюших преобразований является тождество (4.16), которое можно переписать также в виде

$$
k(\varphi(p) \xi)=\varphi([k, p]) \xi+\varphi(p)(k \xi) .
$$

А) Напомним, что

$$
A=\left\langle\xi_{0}, \alpha_{1} \tilde{\xi}_{0}\right\rangle=\left\langle\xi_{0}, \varphi\left(p_{1}\right) \tilde{\xi}_{0}\right\rangle=\left\langle\xi_{0}, \varphi\left(X_{01^{\prime}}\right) \tilde{\xi}_{0}\right\rangle+\left\langle\xi_{0}, \varphi\left(X_{0^{\prime} 1}\right) \tilde{\xi}_{0}\right\rangle
$$

Заметим, что

$$
X_{00}-X_{11}, X_{0^{\prime} 0^{\prime}}-X_{1^{\prime} 1^{\prime}} \in \mathfrak{k}_{1},
$$

следовательно, $X_{0^{\prime} 0^{\prime}} \xi_{0}=X_{1^{\prime} 1^{\prime}} \xi_{0}$ и $X_{00} \tilde{\xi}_{0}=X_{11} \tilde{\xi}_{0}$. Тогда, пользуясь (4.16), получим

$$
\begin{aligned}
\left\langle\xi_{0}, \varphi\left(X_{01^{\prime}}\right) \tilde{\xi}_{0}\right\rangle & =\left\langle\xi_{0},\left(X_{00} \varphi\left(X_{0^{\prime} 1^{\prime}}\right)-\varphi\left(X_{0^{\prime} 1^{\prime}}\right) X_{00}\right) \tilde{\xi}_{0}\right\rangle \\
& =\left\langle\xi_{0}, X_{00} \varphi\left(X_{0^{\prime} 1^{\prime}}\right) \tilde{\xi}_{0}\right\rangle-\left\langle\xi_{0}, \varphi\left(X_{0^{\prime} 1^{\prime}}\right) X_{00} \tilde{\xi}_{0}\right\rangle \\
& =\left\langle X_{0^{\prime} 0^{\prime}} \xi_{0}, \varphi\left(X_{0^{\prime} 1^{\prime}}\right) \tilde{\xi}_{0}\right\rangle-\left\langle\xi_{0}, \varphi\left(X_{0^{\prime} 1^{\prime}}\right) X_{00} \tilde{\xi}_{0}\right\rangle \\
& =\left\langle X_{1^{\prime} 1^{\prime}} \xi_{0}, \varphi\left(X_{0^{\prime} 1^{\prime}}\right) \tilde{\xi}_{0}\right\rangle-\left\langle\xi_{0}, \varphi\left(X_{0^{\prime} 1^{\prime}}\right) X_{11} \tilde{\xi}_{0}\right\rangle \\
& =\left\langle\xi_{0},\left(X_{11} \varphi\left(X_{0^{\prime} 1^{\prime}}\right)-\varphi\left(X_{0^{\prime} 1^{\prime}}\right) X_{11}\right) \tilde{\xi}_{0}\right\rangle=\left\langle\xi_{0}, \varphi\left(X_{0^{\prime} 1}\right) \tilde{\xi}_{0}\right\rangle .
\end{aligned}
$$

Следовательно, $A=2\left\langle\xi_{0}, \varphi\left(X_{01^{\prime}}\right) \tilde{\xi}_{0}\right\rangle$.

Б) Так как $H_{11}+H_{00} \in \mathfrak{k}_{1}$, то $q_{2} \xi_{0}=i\left(H_{11}-H_{00}\right) \xi_{0}=2 i H_{11} \xi_{0}=-2 i H_{00} \xi_{0}$. Тогда

$$
\begin{aligned}
B=\left\langle q_{2} \xi_{0}, \alpha_{2} \tilde{\xi}_{0}\right\rangle & =\left\langle q_{2} \xi_{0}, i \varphi\left(X_{01^{\prime}}-X_{0^{\prime} 1}\right) \tilde{\xi}_{0}\right\rangle \\
& =2\left\langle H_{11} \xi_{0}, \varphi\left(X_{01^{\prime}} \tilde{\xi}_{0}\right)\right\rangle+2\left\langle H_{00} \xi_{0}, \varphi\left(X_{0^{\prime} 1}\right) \tilde{\xi}_{0}\right\rangle .
\end{aligned}
$$

Заметим, что $\left[X_{00}-X_{11}, X_{0^{\prime} 0^{\prime}}+X_{1^{\prime} 1^{\prime}}\right]=H_{00}-H_{11}$. Тогда с учетом $(4.18)$

$$
\begin{aligned}
2\left\langle H_{00} \xi_{0}, \varphi\left(X_{0^{\prime} 1}\right) \tilde{\xi}_{0}\right\rangle & =\left\langle\left(H_{00}-H_{11}\right) \xi_{0}, \varphi\left(X_{0^{\prime} 1}\right) \tilde{\xi}_{0}\right\rangle \\
& =\left\langle\left(X_{00}-X_{11}\right)\left(X_{0^{\prime} 0^{\prime}}+X_{1^{\prime} 1^{\prime}}\right) \xi_{0}, \varphi\left(X_{0^{\prime} 1}\right) \tilde{\xi}_{0}\right\rangle \\
& =\left\langle 2 X_{1^{\prime} 1^{\prime}} \xi_{0},\left(X_{0^{\prime} 0^{\prime}}-X_{1^{\prime} 1^{\prime}}\right) \varphi\left(X_{0^{\prime} 1}\right) \tilde{\xi}_{0}\right\rangle \\
& =-2\left\langle X_{1^{\prime} 1^{\prime}} \xi_{0}, \varphi\left(X_{0^{\prime} 1^{\prime}}\right) \tilde{\xi}_{0}\right\rangle .
\end{aligned}
$$

Аналогично проверяется, что

$$
2\left\langle H_{11} \xi_{0}, \varphi\left(X_{01^{\prime}}\right) \tilde{\xi}_{0}\right\rangle=-2\left\langle X_{1^{\prime} 1^{\prime}} \xi_{0}, \varphi\left(X_{0^{\prime} 1^{\prime}}\right) \tilde{\xi}_{0}\right\rangle
$$

Следовательно, $B=4\left\langle H_{11} \xi_{0}, \varphi\left(X_{01^{\prime}}\right) \tilde{\xi}_{0}\right\rangle$. 
В) По определению

$$
\begin{aligned}
C=\left\langle q_{5} \xi_{0}, \alpha_{5} \tilde{\xi}_{0}\right\rangle= & \left\langle X_{12^{\prime}} \xi_{0}, \varphi\left(X_{02^{\prime}}\right) \tilde{\xi}_{0}\right\rangle+\left\langle X_{12^{\prime}} \xi_{0}, \varphi\left(X_{0^{\prime} 2}\right) \tilde{\xi}_{0}\right\rangle \\
& -\left\langle X_{1^{\prime} 2} \xi_{0}, \varphi\left(X_{02^{\prime}}\right) \tilde{\xi}_{0}\right\rangle-\left\langle X_{1^{\prime} 2} \xi_{0}, \varphi\left(X_{0^{\prime} 2}\right) \tilde{\xi}_{0}\right\rangle .
\end{aligned}
$$

Отметим, что элементы $X_{22}, X_{2^{\prime} 2^{\prime}}$ принадлежат $\mathfrak{k}_{1}$, следовательно, $X_{2^{\prime} 2^{\prime}} \xi_{0}=0$ и $X_{22} \tilde{\xi}_{0}=0$. С учетом этого получим

$$
\begin{aligned}
\left\langle X_{12^{\prime}} \xi_{0}, \varphi\left(X_{02^{\prime}}\right) \tilde{\xi}_{0}\right\rangle & =-\left\langle\left[X_{2^{\prime} 2^{\prime}}, X_{12}\right] \xi_{0}, \varphi\left(X_{02^{\prime}}\right) \tilde{\xi}_{0}\right\rangle \\
& =-\left\langle X_{2^{\prime} 2^{\prime}} X_{12} \xi_{0}, \varphi\left(X_{02^{\prime}}\right) \tilde{\xi}_{0}\right\rangle=-\left\langle X_{12} \xi_{0}, X_{22} \varphi\left(X_{02^{\prime}}\right) \tilde{\xi}_{0}\right\rangle \\
& =\left\langle X_{12} \xi_{0}, \varphi\left(X_{02}\right) \tilde{\xi}_{0}\right\rangle=\left\langle\left[X_{1^{\prime} 2}, X_{11}\right] \xi_{0}, \varphi\left(X_{02}\right) \tilde{\xi}_{0}\right\rangle \\
& =\left\langle X_{1^{\prime} 2} X_{11} \xi_{0}, \varphi\left(X_{02}\right) \tilde{\xi}_{0}\right\rangle-\left\langle X_{1^{\prime} 2} \xi_{0}, X_{1^{\prime} 1^{\prime}} \varphi\left(X_{02}\right) \tilde{\xi}_{0}\right\rangle \\
& =\left\langle X_{1^{\prime} 2} X_{00} \xi_{0}, \varphi\left(X_{02}\right) \tilde{\xi}_{0}\right\rangle-\left\langle X_{1^{\prime} 2} \xi_{0}, \varphi\left(X_{02}\right) X_{1^{\prime} 1^{\prime}} \tilde{\xi}_{0}\right\rangle \\
& =\left\langle X_{1^{\prime} 2} \xi_{0},\left(X_{0^{\prime} 0^{\prime}} \varphi\left(X_{02}-\varphi\left(X_{02}\right) X_{0^{\prime} 0^{\prime}}\right) \tilde{\xi}_{0}\right\rangle\right. \\
& =-\left\langle X_{1^{\prime} 2} \xi_{0}, \varphi\left(X_{0^{\prime} 2}\right) \tilde{\xi}_{0}\right\rangle .
\end{aligned}
$$

Далее заметим, что

$$
\begin{aligned}
\left\langle X_{12^{\prime}} \xi_{0}, \varphi\left(X_{02^{\prime}}\right) \tilde{\xi}_{0}\right\rangle & =-\left\langle\left[X_{2^{\prime} 2^{\prime}}, X_{12}\right] \xi_{0}, \varphi\left(X_{0^{\prime} 2}\right) \tilde{\xi}_{0}\right\rangle \\
& =-\left\langle X_{12} \xi_{0}, X_{22} \varphi\left(X_{0^{\prime} 2}\right) \tilde{\xi}_{0}\right\rangle=-\left\langle X_{12} \xi_{0}, \varphi\left(X_{0^{\prime} 2}\right) X_{22} \tilde{\xi}_{0}\right\rangle=0 .
\end{aligned}
$$

Аналогично $\left\langle X_{1^{\prime} 2} \xi_{0}, \varphi\left(X_{02^{\prime}}\right) \tilde{\xi}_{0}\right\rangle=0$. Следовательно,

$$
C=2\left\langle X_{12^{\prime}} \xi_{0}, \varphi\left(X_{02^{\prime}}\right) \tilde{\xi}_{0}\right\rangle
$$

Пусть $\lambda=(k, k, l, 0, \ldots, 0) \in \Lambda_{0}, \delta_{1}=(1,1,0, \ldots, 0), \delta_{2}=(-1,-1,1,0, \ldots, 0)$. Ненулевые операторы $X \in P_{0}(\lambda, \mu)$ могут быть только при $\mu=\lambda \pm \delta_{1}$ или $\mu=$ $\lambda \pm \delta_{2}$, причем эти операторы определены с точностью до умножения на ненулевое число. Далее мы найдем явньй вид этих операторов.

ПРЕДЛОЖЕНИЕ 4.3. Пусть $\lambda=(k, k, l, 0, \ldots, 0), \quad \mu=\lambda+\delta_{1}$. Cуществует оператор $X_{+}^{(\lambda)} \in P_{0}(\lambda, \mu)$, имеющий вид

$$
\left(X_{+}^{(\lambda)} f\right)(t)=\frac{d f}{d t}(t)-2 k(\operatorname{cth} 2 t) f(t)-2 l(\operatorname{cth} t) f(t) .
$$


ДокАЗАТЕЛЬСтво. А) Пусть $v_{0}$ - старший вектор пространства $E^{\lambda}$. Напомним (см. $\S 2)$, как строится вектор $\xi_{0}$. Элемент $\mathscr{D} \in U(\mathfrak{k})$ определяется формулой (2.18). Пусть

$$
w_{0}=\mathscr{D}^{l} v_{0}, \quad \xi_{0}=\sum_{0 \leqslant r \leqslant k} X_{0^{\prime} 0^{\prime}}^{r} X_{1^{\prime} 1^{\prime}}^{k-r} w_{0}
$$

Тогда $\mathfrak{k}_{1} \xi_{0}=0$ и $\xi_{0} \neq 0$. Можно считать, что вектор $v_{0}$ выбран так, чтобы $\left\langle\xi_{0}, \xi_{0}\right\rangle=1$. Тогда по $(2.28)$

$$
\left\langle w_{0}, w_{0}\right\rangle=(k !(k+1) !)^{-1} .
$$

Аналогично, пусть $\tilde{v}_{0}$ - старший вектор пространства $E^{\mu}, \mu=(k+1, k+1, l, 0, \ldots$ $\ldots, 0)$. Тогда $\widetilde{w}_{0}=\mathscr{D}^{l} \tilde{v}_{0}$ и

$$
\tilde{\xi}_{0}=\sum_{0 \leqslant r \leqslant k+1} X_{0^{\prime} 0^{\prime}}^{r} X_{1^{\prime} 1^{\prime}}^{k-r+1} \widetilde{w}_{0}
$$

при этом можно считать, что $\left\langle\tilde{\xi}_{0}, \tilde{\xi}_{0}\right\rangle=1$.

Пусть $\varphi: \mathfrak{p} \rightarrow \operatorname{Hom}\left(E^{\mu}, E^{\lambda}\right)$ - ненулевой гомоморфизм $\mathfrak{k}$-модулей. Для любого k-модуля $V$ через $V(\delta)$ обозначим весовое пространство веса $\delta$, т.е.

$$
V(\delta)=\{\xi \in V: h \xi=\delta(h) \xi \quad \forall h \in \mathfrak{h}\} .
$$

Будем также говорить, что векторы из $V(\delta)$ имеют вес $\delta$.

Пусть $\omega-$ произвольный вес $\mathfrak{k}$-модуля $\mathfrak{p}, p \in \mathfrak{p}(\omega)$. Тогда $\varphi(p)\left(E^{\mu}(\delta)\right) \subseteq$ $E^{\lambda}(\delta+\omega)$. Действительно, для $\xi \in E^{\mu}(\delta), h \in \mathfrak{h}$ из (4.17) следует, что

$$
h(\varphi(p) \xi)=\varphi([h, p]) \xi+\varphi(p)(h \xi)=(\omega(h)+\delta(h))(\varphi(p) \xi) .
$$

Вектор $X_{0^{\prime} 1^{\prime}}$ является вектором младшего веса $\omega_{0^{\prime} 1^{\prime}}=-\delta_{1}$ в $\mathfrak{p}$. Тогда $\left[X_{\alpha}, X_{0^{\prime} 1^{\prime}}\right]=0$ для любого $\alpha \in R_{-}\left(R_{-}-\right.$множество отрицательных корней алгебры $\mathfrak{k})$ и соответственно

$$
\varphi\left(X_{0^{\prime} 1^{\prime}}\right) X_{\alpha} \xi=X_{\alpha} \varphi\left(X_{0^{\prime} 1^{\prime}} \xi\right) \quad \forall \xi \in E^{\mu}, \quad \forall \alpha \in R_{-} .
$$

Если $\tilde{v}_{0}$ - старший вектор пространства $E^{\mu}$, то

$$
\varphi\left(X_{0^{\prime} 1^{\prime}}\right) \tilde{v}_{0} \subset E^{\lambda}\left(\mu-\delta_{1}\right)=E^{\lambda}(\lambda) .
$$

Если $\varphi\left(X_{0^{\prime} 1^{\prime}}\right) \tilde{v}_{0}=0$, то из $(4.21)$ получим, что $\varphi\left(X_{0^{\prime} 1^{\prime}}\right)\left(X_{\alpha} \tilde{v}_{0}\right)=0$ для любого $\alpha \in R_{-}$и, следовательно, $\varphi\left(X_{0^{\prime} 1^{\prime}}\right)=0$. Тогда из $(4.16)$ следует, что $\varphi\left(\left[k, X_{0^{\prime} 1^{\prime}}\right]\right)=$ 0 для любого $k \in \mathfrak{k}$, а так как $\mathfrak{k}$-модуль $E$ неприводим, то и весь гомоморфизм $\varphi$ равен нулю. Так как $\varphi \neq 0$, то $\varphi\left(X_{0^{\prime} 1^{\prime}}\right) \tilde{v}_{0} \neq 0$, и, умножив $\varphi$ на подходящее число, можно считать, что

$$
\varphi\left(X_{0^{\prime} 1^{\prime}}\right) \tilde{v}_{0}=v_{0}
$$


Так как $\left[H_{23^{\prime}}, X_{0^{\prime} 1^{\prime}}\right]=0$, то $\varphi\left(X_{0^{\prime} 1^{\prime}}\right)\left(H_{23^{\prime}} \xi\right)=H_{23^{\prime}}\left(\varphi\left(X_{0^{\prime} 1^{\prime}}\right) \xi\right)$. Тогда из $(4.21)$ и (2.18) следует, что

$$
\varphi\left(X_{0^{\prime} 1^{\prime}}\right)(\mathscr{D} \xi)=\mathscr{D}\left(\varphi\left(X_{0^{\prime} 1^{\prime}}\right) \xi\right)
$$

и, следовательно,

$$
\varphi\left(X_{0^{\prime} 1^{\prime}}\right) \widetilde{w}_{0}=\varphi\left(X_{0^{\prime} 1^{\prime}}\right) \mathscr{D}^{l} \tilde{v}_{0}=\mathscr{D}^{l} v_{0}=w_{0}
$$

Б) Заметим, что $H_{00} w_{0}=k w_{0}, H_{00} \widetilde{w}_{0}=(k+1) \widetilde{w}_{0}$. Тогда, используя $(4.24)$ и лемму 2.1 , получим

$$
\varphi\left(X_{01^{\prime}}\right) X_{0^{\prime} 0^{\prime}}^{r} \widetilde{w}_{0}=\left(X_{00} \varphi\left(X_{0^{\prime} 1^{\prime}}\right)-\varphi\left(X_{0^{\prime} 1^{\prime}}\right) X_{00}\right) X_{0^{\prime} 0^{\prime}}^{r} \widetilde{w}_{0}=-r X_{0^{\prime} 0^{\prime}}^{r-1} w_{0} .
$$

Следовательно,

$$
\varphi\left(X_{01^{\prime}}\right) \tilde{\xi}_{0}=-\sum_{0 \leqslant r \leqslant k+1} r X_{0^{\prime} 0^{\prime}}^{r-1} X_{1^{\prime} 1^{\prime}}^{k-r+1} w_{0}=-\sum_{0 \leqslant r \leqslant k}(r+1) X_{0^{\prime} 0^{\prime}}^{r} X_{1^{\prime} 1^{\prime}}^{k-r} w_{0}
$$

Найдем скалярное произведение $\left\langle\xi_{0}, \varphi\left(X_{01^{\prime}}\right) \tilde{\xi}_{0}\right\rangle$, учитьвая, что векторы, имеющие различные веса, ортогональны. Воспользовавшись (2.27) и (2.28), получим

$$
\begin{aligned}
A & =2\left\langle\xi_{0}, \varphi\left(X_{01^{\prime}}\right) \tilde{\xi}_{0}\right\rangle=-2 \sum_{0 \leqslant r \leqslant k}\left\langle X_{0^{\prime} 0^{\prime}}^{r} X_{1^{\prime} 1^{\prime}}^{k-r} w_{0}, X_{0^{\prime} 0^{\prime}}^{r} X_{1^{\prime} 1^{\prime}}^{k-r} w_{0}\right\rangle \\
& =-(k !)^{2} 2\left\langle w_{0}, w_{0}\right\rangle \sum_{0 \leqslant r \leqslant k}(r+1)=-k !(k+2) !\left\langle w_{0}, w_{0}\right\rangle=-(k+2) .
\end{aligned}
$$

В) Легко видеть, что $H_{11} X_{0^{\prime} 0^{\prime}}^{r} X_{1^{\prime} 1^{\prime}}^{k-r} w_{0}=(2 r-k) X_{0^{\prime} 0^{\prime}}^{r} X_{1^{\prime} 1^{\prime}}^{k-r} w_{0}$. Тогда

$$
H_{11} \xi_{0}=\sum_{0 \leqslant r \leqslant k}(2 r-k) X_{0^{\prime} 0^{\prime}}^{r} X_{1^{\prime} 1^{\prime}}^{k-r} w_{0}
$$

Используя (4.25) (а также (2.27) и (2.28)), получим

$$
\begin{aligned}
B & =4\left\langle H_{11} \xi_{0}, \varphi\left(X_{01^{\prime}}\right) \tilde{\xi}_{0}\right\rangle=4(k !)^{2}\left\langle w_{0}, w_{0}\right\rangle \sum_{0 \leqslant r \leqslant k}(r+1)(k-2 r) \\
& =-\frac{2}{3} k(k+1)(k+2)(k !)^{2}\left\langle w_{0}, w_{0}\right\rangle=-\frac{2}{3} k(k+2) .
\end{aligned}
$$

Г) Индукцией по $r$ проверим, что

$$
\varphi\left(X_{02^{\prime}}\right) X_{0^{\prime} 0^{\prime}}^{r} \widetilde{w}_{0}=-r X_{0^{\prime} 0^{\prime}}^{r-1} \varphi\left(X_{0^{\prime} 2^{\prime}}\right) \widetilde{w}_{0}
$$

(при $r=0$ считаем, что правая часть равна нулю). 
Пусть $r=0$. Вектор $\varphi\left(X_{02^{\prime}}\right) \widetilde{w}_{0}$ должен иметь вес $\mu+\omega_{02^{\prime}}=\lambda+\omega_{01}+\omega_{02^{\prime}}=$ $\lambda+\omega_{00}+\omega_{12^{\prime}}$. Но ненулевых векторов с таким весом не может быть в пространстве $E^{\lambda}$ со старшим весом $\lambda$.

Пусть (4.26) справедливо для $r-1$. Так как $\left[X_{0^{\prime} 0^{\prime}}, X_{02^{\prime}}\right]=X_{0^{\prime} 2^{\prime}}$ и $\left[X_{0^{\prime} 0^{\prime}}, X_{0^{\prime} 2^{\prime}}\right]=0$, то

$$
\begin{aligned}
\varphi\left(X_{02^{\prime}}\right) X_{0^{\prime} 0^{\prime}}^{r} \widetilde{w}_{0} & =X_{0^{\prime} 0^{\prime}} \varphi\left(X_{02^{\prime}}\right) X_{0^{\prime} 0^{\prime}}^{r-1} \widetilde{w}_{0}-\varphi\left(X_{0^{\prime} 2^{\prime}}\right) X_{0^{\prime} 0^{\prime}}^{r-1} \widetilde{w}_{0} \\
& =-(r-1) X_{0^{\prime} 0^{\prime}}^{r-1} \varphi\left(X_{0^{\prime} 2^{\prime}}\right) \widetilde{w}_{0}-X_{0^{\prime} 0^{\prime}}^{r-1} \varphi\left(X_{0^{\prime} 2^{\prime}}\right) \widetilde{w}_{0} \\
& =-r X_{0^{\prime} 0^{\prime}}^{r-1} \varphi\left(X_{0^{\prime} 2^{\prime}}\right) \widetilde{w}_{0} .
\end{aligned}
$$

Д) По лемме $4.2 C=2\left\langle X_{12^{\prime}} \xi_{0}, \varphi\left(X_{02^{\prime}}\right) \tilde{\xi}_{0}\right\rangle=2\left\langle\xi_{0}, X_{1^{\prime} 2} \varphi\left(X_{02^{\prime}}\right) \tilde{\xi}_{0}\right\rangle$. Из п.Г) следует, что

$$
\begin{aligned}
X_{02^{\prime}} \tilde{\xi}_{0} & =-\sum_{0 \leqslant r \leqslant k+1} r X_{0^{\prime} 0^{\prime}}^{r-1} X_{1^{\prime} 1^{\prime}}^{k-r+1} \varphi\left(X_{0^{\prime} 2^{\prime}}\right) \widetilde{w}_{0} \\
& =-\sum_{0 \leqslant r \leqslant k}(r+1) X_{0^{\prime} 0^{\prime}}^{r} X_{1^{\prime} 1^{\prime}}^{k-r} \varphi\left(X_{0^{\prime} 2^{\prime}}\right) \widetilde{w}_{0} .
\end{aligned}
$$

Тогда

$$
\begin{aligned}
C & =2\left\langle\xi_{0}, X_{1^{\prime} 2} \varphi\left(X_{02^{\prime}}\right) \tilde{\xi}_{0}\right\rangle \\
& =-2 \sum_{0 \leqslant r \leqslant k}(r+1)\left\langle X_{0^{\prime} 0^{\prime}}^{r} X_{1^{\prime} 1^{\prime}}^{k-r} w_{0}, X_{0^{\prime} 0^{\prime}}^{r} X_{1^{\prime} 1^{\prime}}^{k-r} X_{1^{\prime} 2} \varphi\left(X_{0^{\prime} 2^{\prime}}\right) \widetilde{w}_{0}\right\rangle .
\end{aligned}
$$

С учетом (2.27) заметим, что

$$
\begin{aligned}
& \left\langle X_{0^{\prime} 0^{\prime}}^{r} X_{1^{\prime} 1^{\prime}}^{k-r} w_{0}, X_{0^{\prime} 0^{\prime}}^{r} X_{1^{\prime} 1^{\prime}}^{k-r} X_{1^{\prime} 2} \varphi\left(X_{0^{\prime} 2^{\prime}}\right) \widetilde{w}_{0}\right\rangle \\
& \quad=\left\langle X_{00}^{r} X_{0^{\prime} 0^{\prime}}^{r} X_{11}^{k-r} X_{1^{\prime} 1^{\prime}}^{k-r} w_{0}, X_{1^{\prime} 2} \varphi\left(X_{0^{\prime} 2^{\prime}}\right) \widetilde{w}_{0}\right\rangle=(k !)^{2}\left\langle w_{0}, X_{1^{\prime} 2} \varphi\left(X_{0^{\prime} 2^{\prime}}\right) \widetilde{w}_{0}\right\rangle .
\end{aligned}
$$

Тогда из (4.27) получаем, что

$$
C=-k !(k+2) !\left\langle w_{0}, X_{1^{\prime} 2} \varphi\left(X_{0^{\prime} 2^{\prime}}\right) \widetilde{w}_{0}\right\rangle
$$

Е) Напомним, что $w_{0}=\mathscr{D}^{l} v_{0}, \widetilde{w}_{0}=\mathscr{D}^{l} \tilde{v}_{0}$, где $v_{0}, \tilde{v}_{0}-$ старшие векторы в $E^{\lambda}$ и $E^{\mu}$, а $\mathscr{D}$ определено в (2.18). Непосредственно проверяются соотношения

$$
\begin{gathered}
{\left[X_{12^{\prime}}, \mathscr{D}\right]=-X_{2^{\prime} 2^{\prime}}\left(H_{12^{\prime}}+2 H_{23^{\prime}}+2 n-2\right)+X_{1^{\prime} 2^{\prime}} X_{12^{\prime}}} \\
{\left[X_{2^{\prime} 2^{\prime}}, \mathscr{D}\right]=X_{1^{\prime} 2^{\prime}} X_{2^{\prime} 2^{\prime}}}
\end{gathered}
$$

Временно пусть $H=H_{12^{\prime}}+2 H_{23^{\prime}}$; тогда из (4.29) следует, что

$$
X_{12^{\prime}} \mathscr{D}=\left(\mathscr{D}+X_{1^{\prime} 2^{\prime}}\right) X_{12^{\prime}}-X_{2^{\prime} 2^{\prime}}(H+2 n-2) .
$$


Так как $X_{12^{\prime}} v_{0}=0$, то из (4.31) получаем, что

$$
X_{12^{\prime}} \mathscr{D}^{l} v_{0}=-\sum_{1 \leqslant r \leqslant l}\left(\mathscr{D}+X_{1^{\prime} 2^{\prime}}\right)^{r-1} X_{2^{\prime} 2^{\prime}}(H+2 n-2) \mathscr{D}^{l-r} v_{0} .
$$

Заметим, что $(H+2 n-2) \mathscr{D}^{l-r} v_{0}=(k+2 r+2 n-2) \mathscr{D}^{l-r} v_{0}$ и $\left(\mathscr{D}+X_{1^{\prime} 2^{\prime}}\right) X_{2^{\prime} 2^{\prime}}=$ $X_{2^{\prime} 2^{\prime}} \mathscr{D}$ (следует из (4.30)). Тогда из (4.32) получаем

$$
\begin{aligned}
X_{12^{\prime}} w_{0}=X_{12^{\prime}} \mathscr{D}^{l} v_{0} & =-X_{2^{\prime} 2^{\prime}} \mathscr{D}^{l-1} v_{0} \sum_{1 \leqslant r \leqslant l}(k+2 r+2 n-2) \\
& =-l(k+l+2 n-1) X_{2^{\prime} 2^{\prime}} \mathscr{D}^{l-1} v_{0} .
\end{aligned}
$$

Аналогично,

$$
X_{12^{\prime}} \widetilde{w}_{0}=-l(k+l+2 n) X_{2^{\prime} 2^{\prime}} \mathscr{D}^{l-1} \tilde{v}_{0} .
$$

Так как $X_{0^{\prime} 2^{\prime}}=-\left[X_{12^{\prime}}, X_{0^{\prime} 1^{\prime}}\right]$, то

$$
\begin{aligned}
\varphi\left(X_{0^{\prime} 2^{\prime}}\right) \widetilde{w}_{0} & =-\left(X_{12^{\prime}} \varphi\left(X_{0^{\prime} 1^{\prime}}\right)-\varphi\left(X_{0^{\prime} 1^{\prime}}\right) X_{12^{\prime}}\right) \widetilde{w}_{0} \\
& =-X_{12^{\prime}} w_{0}+\varphi\left(X_{0^{\prime} 1^{\prime}}\right) X_{12^{\prime}} \widetilde{w}_{0}=-l X_{2^{\prime} 2^{\prime}} \mathscr{D}^{l-1} v_{0} .
\end{aligned}
$$

Далее заметим, что

$$
X_{1^{\prime} 2} X_{2^{\prime} 2^{\prime}} \mathscr{D}^{l-1} v_{0}=-X_{1^{\prime} 2^{\prime}} \mathscr{D}^{l-1} v_{0}+X_{2^{\prime} 2^{\prime}} X_{1^{\prime} 2} \mathscr{D}^{l-1} v_{0}
$$

и

$$
\left\langle w_{0}, X_{2^{\prime} 2^{\prime}} X_{1^{\prime} 2} \mathscr{D}^{l-1} v_{0}\right\rangle=\left\langle X_{22} w_{0}, X_{1^{\prime} 2} \mathscr{D}^{l-1} v_{0}\right\rangle=0 .
$$

Следовательно,

$$
\left\langle w_{0}, X_{1^{\prime} 2} \varphi\left(X_{0^{\prime} 2^{\prime}}\right) \widetilde{w}_{0}\right\rangle=l\left\langle w_{0}, X_{1^{\prime} 2^{\prime}} \mathscr{D}^{l-1} v_{0}\right\rangle .
$$

Ж) Докажем, что

$$
\left\langle w_{0}, X_{1^{\prime} 2^{\prime}} \mathscr{D}^{l-1} v_{0}\right\rangle=(2 n-2)^{-1}\left\langle w_{0}, w_{0}\right\rangle .
$$

Для этого заметим, что

$$
\left\langle w_{0}, w_{0}\right\rangle=\left\langle w_{0}, \mathscr{D}^{l} v_{0}\right\rangle=\left\langle\mathscr{D}^{*} w_{0}, \mathscr{D}^{l-1} v_{0}\right\rangle,
$$

где $\mathscr{D}^{*}$ задается формулой (2.29), или, после тождественного преобразования,

$$
\mathscr{D}^{*}=\left(H_{23^{\prime}}+2 n-3\right) X_{12}+\sum_{d=3}^{n} X_{1 d} X_{2 d^{\prime}}-\sum_{d=2}^{n} X_{1 d^{\prime}} X_{2 d} .
$$

Так как $X_{2 d^{\prime}} w_{0}=0, X_{2 d} w_{0}=0$ при $d \geqslant 2$ и $H_{23^{\prime}} X_{12} w_{0}=X_{12} w_{0}$, то $\mathscr{D}^{*} w_{0}=(2 n-$ 2) $X_{12} w_{0}$. Тогда

$$
\begin{aligned}
\left\langle w_{0}, w_{0}\right\rangle & =\left\langle\mathscr{D}^{*} w_{0}, \mathscr{D}^{l-1} v_{0}\right\rangle=(2 n-2)\left\langle X_{12} w_{0}, \mathscr{D}^{l-1} v_{0}\right\rangle \\
& =(2 n-2)\left\langle w_{0}, X_{1^{\prime} 2^{\prime}} \mathscr{D}^{l-1} v_{0}\right\rangle .
\end{aligned}
$$

3) Из (4.28), (4.35) и (4.36) следует, что

$$
C=-k !(k+2) ! l(2 n-2)^{-1}\left\langle w_{0}, w_{0}\right\rangle=-(k+2) l(2 n-2)^{-1} .
$$

По гомоморфизму $\varphi$ строится оператор $X \in P_{0}(\lambda, \mu)$, явньй вид которого находится из леммы 4.1 по найденным коэффициентам $A, B, C$. Тогда оператор $X_{+}^{(\lambda)}=\frac{1}{A} X$ имеет вид (4.19), что и доказывает предложение 4.3 . 
ПРЕДЛОЖЕНИЕ 4.4. Пусть $\lambda=(k, k, l, 0, \ldots, 0), \quad k \geqslant 1$. Тогда существует оператор $X_{-}^{(\lambda)} \in P_{0}\left(\lambda, \lambda-\delta_{1}\right)$, имеющий вид

$$
\left(X_{-}^{(\lambda)} f\right)(t)=\frac{d f}{d t}(t)+2(k+2)(\operatorname{cth} 2 t) \cdot f(t)+2(l+2 n-2)(\operatorname{cth} t) \cdot f(t) .
$$

ДоказАТЕЛЬСтво. А) Напомним, что через $E^{\lambda}(\delta)$ обозначается весовое подпространство веса $\delta$ в $E^{\lambda}$. Через $L$ обозначим линейное подпространство, состоящее из векторов $v \in E^{\lambda}\left(\lambda-\omega_{00}-\omega_{11}\right)$, удовлетворяющих условиям $X_{\alpha_{j}} v=0$ при $j=2,3, \ldots, n$ и $X_{-\alpha_{1}}^{k} v=0$, где $\alpha_{1}, \alpha_{2}, \ldots, \alpha_{n}$ - простые корни алгебры $\mathfrak{k}$ (см. (2.10)). Докажем, что $\operatorname{dim} L \leqslant 1$.

Рассмотрим линейное подпространство $L_{1}:=X_{1 n}(L)$. Тогда $L_{1} \subseteq E^{\lambda}(\lambda-$ $\left.\omega_{00}-\omega_{1 n^{\prime}}\right)$. Пусть $v \in L, w=X_{1 n} v \in L_{1}$; тогда $X_{-\alpha_{1}} w=X_{1^{\prime} 2} X_{1 n} v=$ $\left(X_{1 n} X_{1^{\prime} 2}-X_{2 n}\right) v=X_{1 n} X_{1^{\prime} 2} v$. Следовательно, $X_{1^{\prime} 2}^{k} w=X_{1 n} X_{1^{\prime} 2}^{k} v=0$. Любой вектор $\xi \in E^{\lambda}$ может быть представлен в виде $\xi=P(X) v_{0}$, где $P(X)$ некоторый полином от векторов $X_{\alpha}, \alpha \in R_{-}\left(R_{-}-\right.$множество отрицательных корней алгебры $\mathfrak{k}), v_{0}$ - старший вектор. В частности, так как $X_{j^{\prime} r} v_{0}=0$ при $3 \leqslant j<r \leqslant n$, пространство $E^{\lambda}\left(\lambda-\omega_{00}-\omega_{1 n^{\prime}}\right)$ натягивается на векторы $w_{1}=X_{0^{\prime} 0^{\prime}} X_{1^{\prime} n} v_{0}$ и $w_{2}=X_{0^{\prime} 0^{\prime}} X_{1^{\prime} 2} X_{2^{\prime} n} v_{0}$. При $k=0$ вектор $X_{0^{\prime} 0^{\prime}} v_{0}=0$, следовательно, $w_{1}=w_{2}=0$; при $l=0$ вектор $X_{2^{\prime} n} v_{0}=0$, следовательно, $w_{2}=0$. В этих случаях $\operatorname{dim} L_{1} \leqslant 1$. Пусть $k \geqslant 1$ и $l \geqslant 1$. Заметим, что $X_{1^{\prime} 2}^{k} w_{1} \neq 0$, так как

$$
\begin{aligned}
\left\langle X_{1^{\prime} 2}^{k} w_{1}, X_{1^{\prime} 2}^{k} w_{1}\right\rangle & =\left\langle X_{0^{\prime} 0^{\prime}} X_{1^{\prime} n} X_{1^{\prime} 2}^{k} v_{0}, X_{0^{\prime} 0^{\prime}} X_{1^{\prime} n} X_{1^{\prime} 2}^{k} v_{0}\right\rangle \\
& =\left\langle X_{00} X_{0^{\prime} 0^{\prime}} X_{12^{\prime}}^{k} X_{1 n^{\prime}} X_{1^{\prime} n} X_{1^{\prime} 2}^{k} v_{0}, v_{0}\right\rangle=k l(k !)^{2}\left\langle v_{0}, v_{0}\right\rangle \neq 0 .
\end{aligned}
$$

Следовательно, условие $X_{1^{\prime} 2}^{k} w=0$ выделяет в $E^{\lambda}\left(\lambda-\omega_{00}-\omega_{1 n^{\prime}}\right)$ линейное подпространство размерности $\leqslant 1$ и $\operatorname{dim} L_{1} \leqslant 1$.

Предположим, что $\operatorname{dim} L \geqslant 2$. Пусть $v_{1}$ и $v_{2}-$ линейно независимые векторы из $L$. Так как $\operatorname{dim} L_{1} \leqslant 1$, то можно подобрать такую линейную комбинацию $v \neq 0$ этих векторов, что $X_{1 n} v=0$. Заметим, что $X_{3 n} v=0$ и $H_{3 n} v=0$, следовательно, и $X_{3^{\prime} n^{\prime}} v=0$. Тогда $X_{\alpha_{1}+\alpha_{2}} v=X_{13^{\prime}} v=\left[X_{1 n}, X_{3^{\prime} n^{\prime}}\right] v=0$.

Подберем наибольшее число $d_{1} \in \mathbb{Z}_{+}$так, чтобы $X_{\alpha_{1}}^{d_{1}} v \neq 0$; затем возьмем наибольшее $d_{0} \in \mathbb{Z}_{+}$так, что $\xi=X_{\alpha_{0}}^{d_{0}} X_{\alpha_{1}}^{d_{1}} v \neq 0$. Тогда $X_{\alpha_{j}} \xi=0$ при $j=0,1,2, \ldots, n$, следовательно, $\xi$ является старшим вектором. Но вес вектора $\xi$ равен

$$
\lambda-\omega_{00}-\omega_{11}+d_{1} \omega_{12^{\prime}}+d_{0} \omega_{00} \neq \lambda,
$$

что противоречит единственности старшего веса. Следовательно, $v=0$ и $\operatorname{dim} L \leqslant 1$.

Б) Пусть $\varphi: \mathfrak{p} \rightarrow \operatorname{Hom}\left(E^{\mu}, E^{\lambda}\right)$ - гомоморфизм $\mathfrak{k}$-модулей, $\mu=\lambda-\delta_{1}=$ $(k-1, k-1, l, 0, \ldots, 0), \quad k \geqslant 1, v_{0}$ и $\tilde{v}_{0}-$ старшие векторы в $E^{\lambda}$ и $E^{\mu}$ соответственно. Докажем, что для некоторого ненулевого гомоморфизма $\varphi$

$$
\varphi\left(X_{0^{\prime} 1^{\prime}}\right) \tilde{v}_{0}=X_{0^{\prime} 0^{\prime}} \widetilde{\mathscr{D}} v_{0}
$$


где $\widetilde{\mathscr{D}}=(k+1)(k+2 l+2 n-2) X_{1^{\prime} 1^{\prime}}+X_{1^{\prime} 2} \mathscr{D}, \mathscr{D}$ определено в (2.18).

Пусть $\varphi\left(X_{0^{\prime} 1^{\prime}}\right) \tilde{v}_{0}=v$. Повторяя рассуждения п. А) предложения 4.3 , получим, что $v \neq 0$. Вектор $v$ имеет вес $\mu-\delta_{1}=\lambda-\omega_{00}-\omega_{11}$. Так как $\left[X_{\alpha_{j}}, X_{0^{\prime} 1^{\prime}}\right]=0$ для $j \geqslant 2,\left[X_{-\alpha_{1}}, X_{0^{\prime} 1^{\prime}}\right]=0$ и $X_{-\alpha_{1}}^{k} \tilde{v}_{0}$, то $X_{\alpha_{j}} v=0$ для $j \geqslant 2$ и $X_{-\alpha_{1}}^{k} v=0$. Следовательно, вектор $v$ принадлежит подпространству $L$ из п. А). Далее будет показано, что $X_{0^{\prime} 0^{\prime}} \widetilde{\mathscr{D}} v_{0} \in L$ и $X_{0^{\prime} 0^{\prime}} \widetilde{\mathscr{D}} v_{0} \neq 0$. Так как $\operatorname{dim} L \leqslant 1$, то можно считать, что выполняется (4.38).

Проверим, что $X_{0^{\prime} 0^{\prime}} \widetilde{\mathscr{D}} v_{0} \in L$. При $j \geqslant 2\left[X_{\alpha_{j}}, X_{1^{\prime} 1^{\prime}}\right]=0$ и $X_{\alpha_{j}} \mathscr{D} v_{0}=0$ (см. (2.22)), следовательно, и $X_{\alpha_{j}} X_{0^{\prime} 0^{\prime}} \widetilde{\mathscr{D}} v_{0}=0$. Легко видеть, что

$$
\left[X_{-\alpha_{1}}, \mathscr{D}\right]=\left[X_{1^{\prime} 2}, \mathscr{D}\right]=-2 X_{1^{\prime} 1^{\prime}}\left(H_{23^{\prime}}+n-1\right)
$$

Отметим также, что $X_{-\alpha_{1}}^{k+1} v_{0}=0$. Тогда

$$
\begin{aligned}
X_{-\alpha_{1}}^{k+1} \mathscr{D} v_{0} & =-2 \sum_{0 \leqslant r \leqslant k} X_{-\alpha_{1}}^{r} X_{1^{\prime} 1^{\prime}}\left(H_{23^{\prime}}+n-1\right) X_{-\alpha_{1}}^{k-r} v_{0} \\
& =-(k+1)(k+2 l+2 n-2) X_{1^{\prime} 1^{\prime}} X_{-\alpha_{1}}^{k} v_{0} .
\end{aligned}
$$

Следовательно,

$$
X_{-\alpha_{1}}^{k} X_{0^{\prime} 0^{\prime}} \widetilde{\mathscr{D}} v_{0}=X_{0^{\prime} 0^{\prime}}\left((k+1)(k+2 l+2 n-2) X_{1^{\prime} 1^{\prime}} X_{1^{\prime} 2}^{k} v_{0}+X_{1^{\prime} 2}^{k+1} \mathscr{D} v_{0}\right)=0
$$

Нетрудно вычислить, что

$$
\begin{array}{r}
\left\langle X_{0^{\prime} 0^{\prime}} \widetilde{\mathscr{D}} v_{0}, X_{0^{\prime} 0^{\prime}} X_{1^{\prime} 1^{\prime}} v_{0}\right\rangle=\left\langle X_{00} X_{0^{\prime} 0^{\prime}} X_{1^{\prime} 1^{\prime}} \widetilde{\mathscr{D}} v_{0}, v_{0}\right\rangle \\
=k^{2}(k+2 l+2 n-2)(k+l+1)\left\langle v_{0}, v_{0}\right\rangle \neq 0,
\end{array}
$$

так как $k \geqslant 1$. Следовательно, $X_{0^{\prime} 0^{\prime}} \widetilde{\mathscr{D}} v_{0} \neq 0$.

В) Пусть $v_{0}$ и $\widetilde{v}_{0}$ - старшие векторы пространств $E^{\lambda}$ и $E^{\mu}$. Векторы $w_{0}$ и $\xi_{0}$ определяются формулами (4.20). Пусть $\widetilde{w}_{0}=\mathscr{D}^{l} v_{0}$ и

$$
\tilde{\xi}_{0}=\sum_{0 \leqslant r \leqslant k-1} X_{0^{\prime} 0^{\prime}}^{r} X_{1^{\prime} 1^{\prime}}^{k-r-1} \widetilde{w}_{0}
$$

Можно считать, что векторы $v_{0}$ и $\tilde{v}_{0}$ выбраны так, чтобы $\left\langle\xi_{0}, \xi_{0}\right\rangle=1$ и $\left\langle\tilde{\xi}_{0}, \tilde{\xi}_{0}\right\rangle=1$. Тогда (см. $(2.28))\left\langle w_{0}, w_{0}\right\rangle=(k !(k+1) !)^{-1}$. Проводим вычисления аналогично вычислениям из п. Б) предложения 4.3. Получим

$$
\begin{aligned}
\varphi\left(X_{01^{\prime}}\right) \tilde{\xi}_{0} & =X_{00} \varphi\left(X_{0^{\prime} 1^{\prime}}\right) \tilde{\xi}_{0}-\varphi\left(X_{0^{\prime} 1^{\prime}}\right)\left(X_{00} \tilde{\xi}_{0}\right) \\
& =\sum_{0 \leqslant r \leqslant k-1}(k-r) X_{0^{\prime} 0^{\prime}}^{r} X_{1^{\prime} 1^{\prime}}^{k-r-1} \mathscr{D} l \widetilde{D} v_{0} .
\end{aligned}
$$


Далее,

$$
\begin{aligned}
A & =2\left\langle\xi_{0}, \varphi\left(X_{01^{\prime}}\right) \tilde{\xi}_{0}\right\rangle \\
& =2 \sum_{0 \leqslant r \leqslant k-1}(k-r)\left\langle X_{0^{\prime} 0^{\prime}}^{r} X_{1^{\prime} 1^{\prime}}^{k-r} w_{0}, X_{0^{\prime} 0^{\prime}}^{r} X_{1^{\prime} 1^{\prime}}^{k-r-1} \mathscr{D}^{l} \widetilde{\mathscr{D}} v_{0}\right\rangle \\
& =2 \sum_{0 \leqslant r \leqslant k-1}(k-r)\left\langle X_{00}^{r} X_{0^{\prime} 0^{\prime}}^{r} X_{11}^{k-r-1} X_{1^{\prime} 1^{\prime}}^{k-r} w_{0}, \mathscr{D}^{l} \widetilde{\mathscr{D}} v_{0}\right\rangle \\
& =k !(k+1) !\left\langle X_{1^{\prime} 1^{\prime}} w_{0}, \mathscr{D}^{l} \widetilde{\mathscr{D}} v_{0}\right\rangle .
\end{aligned}
$$

Так как вектор $w_{0}$ имеет вес $(k, k, 0, \ldots, 0)$ и $X_{\alpha_{j}} w_{0}=0$ при $j \geqslant 2$, то и $X_{-\alpha_{j}} w_{0}=0$ при $j \geqslant 2$. Тогда $X_{2^{\prime} r} w_{0}=0, X_{2^{\prime} r^{\prime}} w_{0}=0$ при $r \geqslant 2$ и $H_{23^{\prime}} w_{0}=0$, а следовательно, и $\mathscr{D}^{l+1} v_{0}=\mathscr{D} w_{0}=0$. Заметим, что

$$
\left[X_{1^{\prime} 1^{\prime}}, \mathscr{D}\right]=0, \quad\left[X_{1^{\prime} 2}, \mathscr{D}\right]=-X_{1^{\prime} 1^{\prime}}\left(H_{23^{\prime}}+n-1\right)
$$

Тогда

$$
\begin{aligned}
\mathscr{D}^{l} X_{1^{\prime} 2} \mathscr{D} v_{0} & =\left[\mathscr{D}^{l}, X_{1^{\prime} 2}\right] \mathscr{D} v_{0} \\
& =2 X_{1^{\prime} 1^{\prime}}\left(\sum_{0 \leqslant r \leqslant l-1} \mathscr{D}^{l-r-1}\left(H_{23^{\prime}}+n-1\right) \mathscr{D}^{r+1} v_{0}\right) \\
& =l(l+2 n-3) X_{1^{\prime} 1^{\prime}} \mathscr{D}^{l} v_{0}=l(l+2 n-3) X_{1^{\prime} 1^{\prime}} w_{0}
\end{aligned}
$$

И

$$
\begin{aligned}
\mathscr{D}^{l} \widetilde{\mathscr{D}} v_{0} & =(k+1)(k+2 l+2 n-2) X_{1^{\prime} 1^{\prime}} w_{0}+l(l+2 n-3) X_{1^{\prime} 1^{\prime}} w_{0} \\
& =(k+l+1)(k+l+2 n-2) X_{1^{\prime} 1^{\prime}} w_{0} .
\end{aligned}
$$

Следовательно,

$$
\left\langle X_{1^{\prime} 1^{\prime}} w_{0}, \mathscr{D}^{l} \widetilde{\mathscr{D}} v_{0}\right\rangle=\left\langle w_{0}, X_{11} \mathscr{D}^{l} \widetilde{\mathscr{D}} v_{0}\right\rangle=k(k+l+1)(k+l+2 n-2)\left\langle w_{0}, w_{0}\right\rangle
$$

Из (4.41) и (4.44) получим, что

$$
A=k(k+l+1)(k+l+2 n-2) .
$$

Далее, вычисляя, как в п. В) предложения 4.3, с учетом (4.40) и (4.44) находим, что

$$
B=4\left\langle H_{11} \xi_{0}, \varphi\left(X_{01^{\prime}}\right) \tilde{\xi}_{0}\right\rangle=-\frac{2}{3}(k+2) A
$$

Г) Заметим, что

$$
\begin{aligned}
C & =2\left\langle\xi_{0}, X_{1^{\prime} 2} \varphi\left(X_{02^{\prime}}\right) \tilde{\xi}_{0}\right\rangle=2\left\langle\xi_{0}, X_{1^{\prime} 2}\left(X_{00} \varphi\left(X_{0^{\prime} 2^{\prime}}\right)-\varphi\left(X_{0^{\prime} 2^{\prime}}\right) X_{00}\right) \tilde{\xi}_{0}\right\rangle \\
& =2\left\langle\xi_{0}, X_{1^{\prime} 2} X_{00} \varphi\left(X_{0^{\prime} 2^{\prime}}\right) \tilde{\xi}_{0}\right\rangle-2\left\langle\xi_{0}, \varphi\left(X_{0^{\prime} 2^{\prime}}\right) X_{00} \tilde{\xi}_{0}\right\rangle .
\end{aligned}
$$


Используя лемму 2.1, получим, что

$$
\begin{aligned}
\left\langle\xi_{0},\right. & \left.X_{1^{\prime} 2} X_{00} \varphi\left(X_{0^{\prime} 2^{\prime}}\right) \tilde{\xi}_{0}\right\rangle=\left\langle X_{0^{\prime} 0^{\prime}} \xi_{0}, X_{1^{\prime} 2} \varphi\left(X_{0^{\prime} 2^{\prime}}\right) \tilde{\xi}_{0}\right\rangle \\
& =\sum_{0 \leqslant r \leqslant k-1}\left\langle X_{0^{\prime} 0^{\prime}}^{r+1} X_{1^{\prime} 1^{\prime}}^{k-r} w_{0}, X_{1^{\prime} 2} X_{0^{\prime} 0^{\prime}}^{r} X_{1^{\prime} 1^{\prime}}^{k-r-1} \varphi\left(X_{0^{\prime} 2^{\prime}}\right) \widetilde{w}_{0}\right\rangle \\
& =\sum_{0 \leqslant r \leqslant k-1}\left\langle X_{00}^{r} X_{0^{\prime} 0^{\prime}}^{r+1} X_{11}^{k-r-1} X_{1^{\prime} 1^{\prime}}^{k-r} w_{0}, X_{1^{\prime} 2} \varphi\left(X_{0^{\prime} 2^{\prime}}\right) \widetilde{w}_{0}\right\rangle \\
& =((k-1) !)^{2}\left\langle X_{0^{\prime} 0^{\prime}} X_{1^{\prime} 1^{\prime}} w_{0}, X_{1^{\prime} 2} \varphi\left(X_{0^{\prime} 2^{\prime}}\right) \widetilde{w}_{0}\right\rangle \sum_{0 \leqslant r \leqslant k-1}(r+1)(k-r) .
\end{aligned}
$$

Аналогично,

$$
\begin{aligned}
& \left\langle\xi_{0}, X_{1^{\prime} 2} \varphi\left(X_{0^{\prime} 2^{\prime}}\right) X_{00} \tilde{\xi}_{0}\right\rangle \\
& \quad=((k-1) !)^{2}\left\langle X_{0^{\prime} 0^{\prime}} X_{1^{\prime} 1^{\prime}} w_{0}, X_{1^{\prime} 2} \varphi\left(X_{0^{\prime} 2^{\prime}}\right) \widetilde{w}_{0}\right\rangle \sum_{0 \leqslant r \leqslant k-1} r(k-r) .
\end{aligned}
$$

Тогда

$$
\begin{aligned}
C & =2((k-1) !)^{2}\left\langle X_{0^{\prime} 0^{\prime}} X_{1^{\prime} 1^{\prime}} w_{0}, X_{1^{\prime} 2} \varphi\left(X_{0^{\prime} 2^{\prime}}\right) \widetilde{w}_{0}\right\rangle \sum_{0 \leqslant r \leqslant k-1}(k-r) \\
& =(k-1) !(k+1) !\left\langle X_{0^{\prime} 0^{\prime}} X_{1^{\prime} 1^{\prime}} w_{0}, X_{1^{\prime} 2} \varphi\left(X_{0^{\prime} 2^{\prime}}\right) \widetilde{w}_{0}\right\rangle .
\end{aligned}
$$

Taк как $X_{0^{\prime} 2^{\prime}}=-\left[X_{12^{\prime}}, X_{0^{\prime} 1^{\prime}}\right]$, то

$$
\begin{aligned}
& \left\langle X_{0^{\prime} 0^{\prime}} X_{1^{\prime} 1^{\prime}} w_{0}, X_{1^{\prime} 2} \varphi\left(X_{0^{\prime} 2^{\prime}}\right) \widetilde{w}_{0}\right\rangle=\left\langle X_{0^{\prime} 0^{\prime}} X_{1^{\prime} 1^{\prime}} w_{0}, X_{1^{\prime} 2} \varphi\left(X_{0^{\prime} 1^{\prime}}\right) X_{12^{\prime}} \widetilde{w}_{0}\right\rangle \\
& -\left\langle X_{0^{\prime} 0^{\prime}} X_{1^{\prime} 1^{\prime}} w_{0}, X_{1^{\prime} 2} X_{12^{\prime}} \varphi\left(X_{0^{\prime} 1^{\prime}}\right) \widetilde{w}_{0}\right\rangle .
\end{aligned}
$$

Используя (4.38), (4.43) и (4.33), получим, что

$$
\begin{aligned}
\varphi\left(X_{0^{\prime} 1^{\prime}}\right) \widetilde{w}_{0} & =\varphi\left(X_{0^{\prime} 1^{\prime}}\right) \mathscr{D}^{l} \widetilde{v}_{0}=X_{0^{\prime} 0^{\prime}} \mathscr{D}^{l} \widetilde{\mathscr{D}} v_{0} \\
& =(k+l+1)(k+l+2 n-2) X_{0^{\prime} 0^{\prime}} X_{1^{\prime} 1^{\prime}} w_{0}, \\
X_{12^{\prime}} X_{1^{\prime} 1^{\prime}} w_{0} & =-X_{1^{\prime} 2} w_{0}+X_{1^{\prime} 1^{\prime}} X_{12^{\prime}} w_{0} \\
& =-X_{12^{\prime}} w_{0}-l(k+l+2 n-1) X_{2^{\prime} 2^{\prime}} \mathscr{D}^{l-1} v_{0}
\end{aligned}
$$

и

$$
\begin{aligned}
\varphi\left(X_{0^{\prime} 1^{\prime}}\right) X_{12^{\prime}} \widetilde{w}_{0} & =-l(k+l+2 n-2) \varphi\left(X_{0^{\prime} 1^{\prime}}\right) X_{2^{\prime} 2^{\prime}} \mathscr{D}^{l-1} \tilde{v}_{0} \\
& =-l(k+l+2 n-2) X_{2^{\prime} 2^{\prime}} \mathscr{D}^{l-1} \widetilde{\mathscr{D}} v_{0} .
\end{aligned}
$$

Из (4.47)-(4.49) после несложных преобразований с использованием формулы (4.36) вычисляются слагаемые в правой части (4.46). Окончательно получаем $C=-k(k+l+1)(k+l+2 n-2)(l+2 n-2)(2 n-2)^{-1}=-(l+2 n-2)(2 n-2)^{-1} A$.

По гомоморфизму $\varphi$ строится оператор $X \in P_{0}(\lambda, \mu)$, явный вид которого находится по лемме 4.1 и найденным коэффициентам $A, B, C$. Тогда оператор $X_{-}^{(\lambda)}=$ $\frac{1}{A} X$ имеет вид (4.37). 
ПРЕДЛОЖЕНИЕ 4.5. Пусть $\lambda=(k, k, l, 0, \ldots, 0), \quad l \geqslant 1$. Тогда существует оператор $Y_{-}^{(\lambda)} \in P_{0}\left(\lambda, \lambda-\delta_{2}\right)$, имеющий вид

$$
\left(Y_{-}^{(\lambda)} f\right)(t)=\frac{d f}{d t}(t)-2 k(\operatorname{cth} 2 t) f(t)+2(k+l+2 n-1)(\operatorname{cth} t) f(t) .
$$

ДокАЗАТЕЛЬСтво. А) В нашем случае $\mu=\lambda-\delta_{2}=\lambda+\omega_{02^{\prime}}=(k+1, k+1$, $l-1,0, \ldots, 0)$. Как и в предыдущих предложениях, пусть $v_{0}$ и $\tilde{v}_{0}-$ старшие векторы в $E^{\lambda}$ и $E^{\mu}$, векторы $w_{0}$ и $\xi_{0}$ определяются формулами (4.20),

$$
\widetilde{w}_{0}=\mathscr{D}^{l-1} \tilde{v}_{0}, \quad \tilde{\xi}_{0}=\sum_{0 \leqslant r \leqslant k+1} X_{0^{\prime} 0^{\prime}}^{r} X_{1^{\prime} 1^{\prime}}^{k-r+1} \widetilde{w}_{0} .
$$

Докажем, что для некоторого ненулевого гомоморфизма $\varphi$

$$
\varphi\left(X_{0^{\prime} 1^{\prime}}\right) \tilde{v}_{0}=\mathscr{D} v_{0}
$$

Пусть $v=\varphi\left(X_{0^{\prime} 1^{\prime}}\right) \tilde{v}_{0}$. Повторяя рассуждения п. А) доказательства предложения 4.3 , получим, что $v \neq 0$. Вектор $v \in E^{\lambda}\left(\lambda-\omega_{12}\right)$, и очевидно, что $X_{\alpha_{j}} v=0$ для $j=2,3, \ldots, n$. Временно обозначим через $L$ подпространство, состояшее из всех векторов $v \in E^{\lambda}\left(\lambda-\omega_{12}\right)$, удовлетворяющих условиям $X_{\alpha_{j}} v=0$ при $j=2,3, \ldots, n$. Тогда вектор $\mathscr{D} v_{0} \in L$ и $\mathscr{D} v_{0} \neq 0$, так как $w_{0}=\mathscr{D}^{l} v_{0} \neq 0$. Если доказать, что $\operatorname{dim} L \leqslant 1$, то, умножив $\varphi$ на подходяшее число, можно добиться выполнения равенства (4.51).

Пусть $L_{1}=X_{1 n}(L)$; тогда $L_{1} \subseteq E^{\lambda}\left(\lambda-\omega_{2 n^{\prime}}\right)$ и, следовательно, $\operatorname{dim} L_{1} \leqslant$ $\operatorname{dim} E^{\lambda}\left(\lambda-\omega_{2 n^{\prime}}\right)=1$. Далее доказательство неравенства $\operatorname{dim} L \leqslant 1$ получается повторением рассуждений из п. А) предложения 4.4.

Б) Из (4.51) следует, что

$$
\varphi\left(X_{0^{\prime} 1^{\prime}}\right) \widetilde{w}_{0}=\varphi\left(X_{0^{\prime} 1^{\prime}}\right) \mathscr{D}^{l-1} \tilde{v}_{0}=\mathscr{D}^{l-1} \varphi\left(X_{0^{\prime} 1^{\prime}}\right) \tilde{v}_{0}=\mathscr{D}^{l} v_{0}=w_{0} .
$$

Почти дословно повторяя вычисления пп. Б) и В) предложения 4.3 , получаем, что $A=-(k+2), B=-\frac{2}{3} k(k+2)$. Вычисление коэффициента $C$ проводится, как в п. Г) предложения 4.4, и не содержит принципиальных трудностей. Получаем, что

$$
C=(k+2)(k+l+2 n-1)(2 n-2)^{-1} .
$$

По гомоморфизму $\varphi$ строим оператор $X \in P_{0}(\lambda, \mu)$; тогда оператор $Y_{+}^{(\lambda)}=\frac{1}{A} X$ имеет вид (4.50).

ПРЕДЛОЖЕНИЕ 4.6. Пусть $\lambda=(k, k, l, 0, \ldots, 0), \quad k \geqslant 1$. Тогда существует оператор $Y_{+}^{(\lambda)} \in P_{0}\left(\lambda, \lambda+\delta_{2}\right)$, имеющий вид

$$
\left(Y_{+}^{(\lambda)} f\right)(t)=\frac{d f}{d t}(t)+2(k+2)(\operatorname{cth} 2 t) f(t)-2(k+l+1)(\operatorname{cth} t) f(t) .
$$


ДокАЗАТЕЛЬСТво. А) В рассматриваемом случае $\mu=\lambda+\delta_{2}=(k-1, k-1$, $l+1,0, \ldots, 0)$. Векторы $v_{0}, w_{0}, \xi_{0} \in E^{\lambda}$ те же, что и в предыдущих предложениях, $\tilde{v}_{0}-$ старший вектор в $E^{\mu}, \widetilde{w}_{0}=\mathscr{D}^{l+1} \tilde{v}_{0}$,

$$
\tilde{\xi}_{0}=\sum_{0 \leqslant r \leqslant k-1} X_{0^{\prime} 0^{\prime}}^{r} X_{1^{\prime} 1^{\prime}}^{k-r-1} \widetilde{w}_{0}
$$

Если $\varphi$ - ненулевой гомоморфизм $\mathfrak{k}$-модулей из $\mathfrak{p}$ в $\operatorname{Hom}\left(E^{\mu}, E^{\lambda}\right)$, то вектор $\varphi\left(X_{0^{\prime} 1^{\prime}}\right) \tilde{v}_{0}$ не равен нулю и имеет вес $\mu-\omega_{01}=\lambda-\omega_{00}-\omega_{12^{\prime}}$. Но пространство $E^{\lambda}\left(\lambda-\omega_{00}-\omega_{12^{\prime}}\right)$ одномерно и порождается вектором $X_{0^{\prime} 0^{\prime}} X_{1^{\prime} 2} v_{0}$. Поэтому, умножив $\varphi$ на подходящее число, можно считать, что

$$
\varphi\left(X_{0^{\prime} 1^{\prime}}\right) \tilde{v}_{0}=X_{0^{\prime} 0^{\prime}} X_{1^{\prime} 2} v_{0}
$$

Б) Напомним, что $\mathscr{D}^{l+1} v_{0}=0$ (см. п.В) предложения 4.4) и $\left[X_{1^{\prime} 2}, \mathscr{D}\right]=$ $-2 X_{1^{\prime} 1^{\prime}}\left(H_{23^{\prime}}+n-1\right)$. Тогда

$\mathscr{D}^{l+1} X_{1^{\prime} 2} v_{0}=2 X_{1^{\prime} 1^{\prime}} \sum_{0 \leqslant r \leqslant l} \mathscr{D}^{r}\left(H_{23^{\prime}}+n-1\right) \mathscr{D}^{l-r} v_{0}=(l+1)(l+2 n-2) X_{1^{\prime} 1^{\prime}} \mathscr{D}^{l} v_{0}$.

Следовательно,

$\varphi\left(X_{0^{\prime} 1^{\prime}}\right) \widetilde{w}_{0}=\varphi\left(X_{0^{\prime} 1^{\prime}}\right) \mathscr{D}^{l+1} \tilde{v}_{0}=X_{0^{\prime} 0^{\prime}} \mathscr{D}^{l+1} X_{1^{\prime} 2} v_{0}=(l+1)(l+2 n-2) X_{0^{\prime} 0^{\prime}} X_{1^{\prime} 1^{\prime}} w_{0}$.

Дальнейшее вычисление коэффициентов $A, B, C$ проводится так же, как в предложении 4.4, и не содержит принципиальных трудностей. Получаем

$$
A=k(l+1)(l+2 n-2), \quad B=-\frac{2}{3}(k+2) A, \quad C=(k+l+1)(2 n-2)^{-1} A .
$$

По гомоморфизму $\varphi$ строится оператор $X \in P_{0}(\lambda, \mu)$, тогда оператор $Y_{-}^{(\lambda)}=\frac{1}{A} X$ имеет вид (4.53).

Пользуясь явным видом операторов $\tilde{\Delta}, X_{ \pm}^{(\lambda)}, Y_{ \pm}^{(\lambda)}$ (см. (3.12), (4.19), (4.37), (4.50), (4.53)), непосредственным вычислением проверяются соотношения

$$
\begin{aligned}
& X_{-}^{\left(\lambda+\delta_{1}\right)} X_{+}^{(\lambda)} f=\tilde{\Delta} f-4(k+l)(k+l+2 n+1) f, \\
& X_{+}^{\left(\lambda-\delta_{1}\right)} X_{-}^{(\lambda)} f=\tilde{\Delta} f-4(k+l-1)(k+l+2 n) f, \\
& Y_{+}^{\left(\lambda-\delta_{2}\right)} Y_{-}^{(\lambda)} f=\tilde{\Delta} f-4(l-2)(l+2 n-1) f, \\
& Y_{-}^{\left(\lambda+\delta_{2}\right)} Y_{+}^{(\lambda)} f=\tilde{\Delta} f-4(l-1)(l+2 n) f .
\end{aligned}
$$

Доказательство теорем I и II проводится аналогично доказательству соответствующих теорем в [1], поэтому отметим только основные моменты доказательств. Доказательство теоремы І отдельно проводится для случая $\lambda=(0, \ldots, 0)$ и затем 
уже в общем случае. Заметим, что при $\lambda=0$ оператор $\tilde{\Delta}$ совпадает с дифференциальным оператором

$$
\Delta_{d, r}=\frac{d^{2}}{t^{2}}+(2 k+2 r+1) \operatorname{cth} t \frac{d}{d t}+(2 r+1) \operatorname{th} t \frac{d}{d t}
$$

при $d=2 n-2, r=1$, который рассматривался в $[1, \S 6]$. Дальнейшие шаги доказательства теоремы І при $\lambda=0$ проходят и для нашего случая $M=\operatorname{Sp}(1, n) / \operatorname{Sp}(1) \times$ $\operatorname{Sp}(n)$, если при этом считать, что $\Phi=\Phi_{2 n-2,1}\left(\Phi_{d, r}-\right.$ преобразование из леммы 5.2 в [1]).

В общем случае, при $\lambda=(k, k, l, 0, \ldots, 0), k, l \geqslant 0$, доказательство теоремы $\mathbf{I}$ проводится индукцией по $p=k+2 l$. При $p=0$ (тогда $\lambda=0$ ) теорема доказана. Используя явньй вид операторов $X_{ \pm}^{(\lambda)}, Y_{ \pm}^{(\lambda)}$ и соотношения (4.54)-(4.57) и рассуждая, как при доказательстве леммы 6.12 в [1], доказываем следующую лемму.

Лемма 4.7. Справедливы следующие утверждения:

1) $\operatorname{dim} V_{\mu, r}^{(\lambda)}=r$

2) $\operatorname{Ker} X_{-}^{(\lambda)}=\{0\}, \quad \operatorname{Ker} Y_{-}^{(\lambda)}=\{0\}$

3) $\operatorname{Ker} X_{+}^{(\lambda)}=V_{i(2 k+2 l+2 n+1), 1}^{(\lambda)}, \quad \operatorname{Ker} Y_{+}^{(\lambda)}=V_{i(2 l+2 n-1), 1}^{(\lambda)}$;

4) $X_{-}^{(\lambda)}\left(V_{\mu, r}^{(\lambda)}\right)=V_{\mu, r}^{\left(\lambda-\delta_{1}\right)}, Y_{-}^{(\lambda)}\left(V_{\mu, r}^{(\lambda)}\right)=V_{\mu, r}^{\left(\lambda-\delta_{2}\right)}$;

5) $X_{+}^{(\lambda)}\left(V_{\mu, r}^{(\lambda)}\right)=V_{\mu, r_{1}}^{\left(\lambda+\delta_{1}\right)}$, əде $r_{1}=r$ nрu $\mu \neq i(2 k+2 l+2 n+1) u r_{1}=r-1$ при $\mu=i(2 k+2 l+2 n+1)$;

6) $Y_{+}^{(\lambda)}\left(V_{\mu, r}^{(\lambda)}\right)=V_{\mu, r_{2}}^{\left(\lambda+\delta_{2}\right)}$, əде $r_{2}=r$ npu $\mu \neq i(2 l+2 n-1) u r_{2}=r-1 n p u$ $\mu=i(2 l+2 n-1)$.

С использованием этой леммы оставшаяся часть доказательства теоремы I проводится так же, как при доказательстве соответствуюшей теоремы в [1]. Доказательство теоремы II проводится так же, как в [1], без существенных отличий.

\section{Список литературы}

1. Платонов C.C. Инвариантные подпространства в некоторых функциональных пространствах на симметрических пространствах // Изв. АН. Сер. матем. 1995. Т. 59. № 5. C. $127-172$.

2. Berenstein C. A. Spectral synthesis on symmetric spaces // Contemp. Math. 1987. V. 63. P. $1-25$.

3. Berenstein C.A., Gay R. Sur la synthése spectrale dans les espaces symmetriques // J. Math. Pure et Appl. 1986. V. 65. P. 323-334.

4. Wawrzynczyk A. Spectral analisis and synthesis on symmetric spaces // J. Math. Ann. and Appl. 1987. V. 127. P. 1-17.

5. Кучмент П. А. О спектральном синтезе в пространствах решений дифференциальных уравнений, инвариантных относительно групп преобразований // Применение топологии в современном анализе. Воронеж: Изд-во ВГУ, 1985. С. 87-105.

6. Кучмент П. А. Сферическое представление решений инвариантных дифференциальных уравнений на римановом симметрическом пространстве некомпактного типа // Изв. АН СССР. Сер. матем. 1985. Т. 49. № 4. С. 1260-1273. 
7. Кучмент П. А. О периодических в среднем функциях на симметрических пространствах // Функцион. анализ и его прилож. 1982. Т. 16. № 3. С. 68-69.

8. Хелгасон $C$. Дифференциальная геометрия и симметрические пространства. М.: Мир, 1964.

9. Робертсон А., Робертсон Б. Топологические векторные пространства. М.: Мир, 1967.

10. Платонов C.C. Инвариантные подпространства в некоторых функциональных пространствах на $n$-мерном пространстве Лобачевского // Матем. сб. 1988. Т. 137. № 12 . C. $435-461$.

11. Платонов C.C. О спектральном синтезе на симметрических пространствах ранга 1 // Алгебра и анализ. 1992. Т. 4. №4. С. 174-187.

12. Гото М., Гросханс Ф. Полупростые алгебры Ли. М.: Мир, 1981.

13. Бурбаки Н. Группы и алгебры Ли. Гл. VII, VIII. М.: Мир, 1978.

14. Helgason S. A duality for symmetric spaces with applications to group representations. I // Adv. Math. 1970. V. 5. P. 1-154.

15. Платонов C.C. Инвариантные подпространства в некоторых функциональных пространствах на симметрических пространствах ранга 1 (симплектический и особый случаи) // ДАН СССР. 1992. Т. 322. № 2. С. 243-247.

16. Kass N. Explicit decompositions of some tensor products of modules for semisimple Lie algebras // Commun. Algebra. 1987. V. 15. P. $2251-2261$.

17. Хелгасон C. Группы и геометрический анализ. М.: Мир, 1987.

Петрозаводский государственный университет.

Поступило в редакцию

E-mail: platonov@mainpgu.karelia.ru

25.I.1996 